DESY-07-126

August 2007

\title{
Dijet production in diffractive deep inelastic scattering at HERA
}

ZEUS Collaboration

\begin{abstract}
The production of dijets in diffractive deep inelastic scattering has been measured with the ZEUS detector at HERA using an integrated luminosity of $61 \mathrm{pb}^{-1}$. The dijet cross section has been measured for virtualities of the exchanged virtual photon, $5<Q^{2}<100 \mathrm{GeV}^{2}$, and $\gamma^{*} p$ centre-of-mass energies, $100<W<250$ $\mathrm{GeV}$. The jets, identified using the inclusive $k_{T}$ algorithm in the $\gamma^{*} p$ frame, were required to have a transverse energy $E_{T, \text { jet }}^{*}>4 \mathrm{GeV}$ and the jet with the highest transverse energy was required to have $E_{T \text {,jet }}^{*}>5 \mathrm{GeV}$. All jets were required to be in the pseudorapidity range $-3.5<\eta_{\text {jet }}^{*}<0$. The differential cross sections are compared to leading-order predictions and next-to-leadingorder QCD calculations based on recent diffractive parton densities extracted from inclusive diffractive deep inelastic scattering data.
\end{abstract}




\section{The ZEUS Collaboration}

S. Chekanov ${ }^{1}$, M. Derrick, S. Magill, B. Musgrave, D. Nicholass ${ }^{2}$, J. Repond, R. Yoshida Argonne National Laboratory, Argonne, Illinois 60439-4815, USA ${ }^{n}$

M.C.K. Mattingly

Andrews University, Berrien Springs, Michigan 49104-0380, USA

M. Jechow, N. Pavel ${ }^{\dagger}$, A.G. Yagües Molina

Institut für Physik der Humboldt-Universität zu Berlin, Berlin, Germany ${ }^{b}$

S. Antonelli, P. Antonioli, G. Bari, M. Basile, L. Bellagamba, M. Bindi, D. Boscherini,

A. Bruni, G. Bruni, L. Cifarelli, F. Cindolo, A. Contin, M. Corradi, S. De Pasquale,

G. Iacobucci, A. Margotti, R. Nania, A. Polini, G. Sartorelli, A. Zichichi

University and INFN Bologna, Bologna, Italy $^{e}$

D. Bartsch, I. Brock, H. Hartmann, E. Hilger, H.-P. Jakob, M. Jüngst, O.M. Kind ${ }^{3}$, A.E. Nuncio-Quiroz, E. Paul ${ }^{4}$, R. Renner ${ }^{5}$, U. Samson, V. Schönberg, R. Shehzadi, M. Wlasenko Physikalisches Institut der Universität Bonn, Bonn, Germany ${ }^{b}$

N.H. Brook, G.P. Heath, J.D. Morris

H.H. Wills Physics Laboratory, University of Bristol, Bristol, United Kingdom ${ }^{m}$

M. Capua, S. Fazio, A. Mastroberardino, M. Schioppa, G. Susinno, E. Tassi

Calabria University, Physics Department and INFN, Cosenza, Italy ${ }^{e}$

J.Y. $\mathrm{Kim}^{6}$, K.J. Ma ${ }^{7}$

Chonnam National University, Kwangju, South Korea ${ }^{g}$

Z.A. Ibrahim, B. Kamaluddin, W.A.T. Wan Abdullah

Jabatan Fizik, Universiti Malaya, 50603 Kuala Lumpur, Malaysia ${ }^{r}$

Y. Ning, Z. Ren, F. Sciulli

Nevis Laboratories, Columbia University, Irvington on Hudson, New York 10027o

J. Chwastowski, A. Eskreys, J. Figiel, A. Galas, M. Gil, K. Olkiewicz, P. Stopa, L. Zawiejski

The Henryk Niewodniczanski Institute of Nuclear Physics, Polish Academy of Sciences, Cracow, Poland ${ }^{i}$

L. Adamczyk, T. Bołd, I. Grabowska-Bołd, D. Kisielewska, J. Łukasik, M. Przybycień,

L. Suszycki

Faculty of Physics and Applied Computer Science, AGH-University of Science and Technology, Cracow, Poland ${ }^{p}$

A. Kotański ${ }^{8}$, W. Słomiński ${ }^{9}$

Department of Physics, Jagellonian University, Cracow, Poland 
V. Adler ${ }^{10}$, U. Behrens, I. Bloch, C. Blohm, A. Bonato, K. Borras, R. Ciesielski, N. Coppola, A. Dossanov, V. Drugakov, J. Fourletova, A. Geiser, D. Gladkov, P. Göttlicher ${ }^{11}$, J. Grebenyuk, I. Gregor, T. Haas, W. Hain, C. Horn ${ }^{12}$, A. Hüttmann, B. Kahle, I.I. Katkov, U. Klein ${ }^{13}$, U. Kötz, H. Kowalski, E. Lobodzinska, B. Löhr, R. Mankel, I.-A. MelzerPellmann, S. Miglioranzi, A. Montanari, T. Namsoo, D. Notz, L. Rinaldi, P. Roloff, I. Rubinsky, R. Santamarta, U. Schneekloth, A. Spiridonov ${ }^{14}$, H. Stadie, D. Szuba ${ }^{15}$, J. Szuba ${ }^{16}$, T. Theedt, G. Wolf, K. Wrona, C. Youngman, W. Zeuner

Deutsches Elektronen-Synchrotron DESY, Hamburg, Germany

W. Lohmann, S. Schlenstedt

Deutsches Elektronen-Synchrotron DESY, Zeuthen, Germany

G. Barbagli, E. Gallo, P. G. Pelfer

University and INFN Florence, Florence, Italy ${ }^{e}$

A. Bamberger, D. Dobur, F. Karstens, N.N. Vlasov ${ }^{17}$

Fakultät für Physik der Universität Freiburg i.Br., Freiburg i.Br., Germany ${ }^{b}$

P.J. Bussey, A.T. Doyle, W. Dunne, M. Forrest, D.H. Saxon, I.O. Skillicorn

Department of Physics and Astronomy, University of Glasgow, Glasgow, United Kingdom $^{m}$

I. Gialas ${ }^{18}$, K. Papageorgiu

Department of Engineering in Management and Finance, Univ. of Aegean, Greece

T. Gosau, U. Holm, R. Klanner, E. Lohrmann, H. Salehi, P. Schleper, T. Schörner-Sadenius,

J. Sztuk, K. Wichmann, K. Wick

Hamburg University, Institute of Exp. Physics, Hamburg, Germany ${ }^{b}$

C. Foudas, C. Fry, K.R. Long, A.D. Tapper

Imperial College London, High Energy Nuclear Physics Group, London, United Kingdom ${ }^{m}$

M. Kataoka ${ }^{19}$, T. Matsumoto, K. Nagano, K. Tokushuku ${ }^{20}$, S. Yamada, Y. Yamazaki ${ }^{21}$ Institute of Particle and Nuclear Studies, KEK, Tsukuba, Japan ${ }^{f}$

A.N. Barakbaev, E.G. Boos, N.S. Pokrovskiy, B.O. Zhautykov

Institute of Physics and Technology of Ministry of Education and Science of Kazakhstan, Almaty, Kazakhstan

V. Aushev ${ }^{1}$, M. Borodin, A. Kozulia, M. Lisovyi

Institute for Nuclear Research, National Academy of Sciences, Kiev and Kiev National University, Kiev, Ukraine

D. Son

Kyungpook National University, Center for High Energy Physics, Daegu, South Korea ${ }^{g}$ 
J. de Favereau, K. Piotrzkowski

Institut de Physique Nucléaire, Université Catholique de Louvain, Louvain-la-Neuve, Belgium $^{q}$

F. Barreiro, C. Glasman², M. Jimenez, L. Labarga, J. del Peso, E. Ron, M. Soares, J. Terrón, M. Zambrana

Departamento de Física Teórica, Universidad Autónoma de Madrid, Madrid, Spain ${ }^{l}$

F. Corriveau, C. Liu, R. Walsh, C. Zhou

Department of Physics, McGill University, Montréal, Québec, Canada H3A 2T8 a

T. Tsurugai

Meiji Gakuin University, Faculty of General Education, Yokohama, Japan ${ }^{f}$

A. Antonov, B.A. Dolgoshein, V. Sosnovtsev, A. Stifutkin, S. Suchkov

Moscow Engineering Physics Institute, Moscow, Russia ${ }^{j}$

R.K. Dementiev, P.F. Ermolov, L.K. Gladilin, L.A. Khein, I.A. Korzhavina, V.A. Kuzmin, B.B. Levchenko ${ }^{23}$, O.Yu. Lukina, A.S. Proskuryakov, L.M. Shcheglova, D.S. Zotkin, S.A. Zotkin

Moscow State University, Institute of Nuclear Physics, Moscow, Russia ${ }^{k}$

I. Abt, C. Büttner, A. Caldwell, D. Kollar, W.B. Schmidke, J. Sutiak

Max-Planck-Institut für Physik, München, Germany

G. Grigorescu, A. Keramidas, E. Koffeman, P. Kooijman, A. Pellegrino, H. Tiecke, M. Vázquez ${ }^{19}$, L. Wiggers

NIKHEF and University of Amsterdam, Amsterdam, Netherlands ${ }^{h}$

N. Brümmer, B. Bylsma, L.S. Durkin, A. Lee, T.Y. Ling

Physics Department, Ohio State University, Columbus, Ohio $43210^{n}$

P.D. Allfrey, M.A. Bell, A.M. Cooper-Sarkar, R.C.E. Devenish, J. Ferrando, B. Foster, K. Korcsak-Gorzo, K. Oliver, S. Patel, V. Roberfroid ${ }^{24}$, A. Robertson, P.B. Straub, C. Uribe-Estrada, R. Walczak

Department of Physics, University of Oxford, Oxford United Kingdom ${ }^{m}$

P. Bellan, A. Bertolin, R. Brugnera, R. Carlin, F. Dal Corso, S. Dusini, A. Garfagnini, S. Limentani, A. Longhin, L. Stanco, M. Turcato

Dipartimento di Fisica dell' Università and INFN, Padova, Italy ${ }^{e}$

B.Y. Oh, A. Raval, J. Ukleja ${ }^{25}$, J.J. Whitmore ${ }^{26}$

Department of Physics, Pennsylvania State University, University Park, Pennsylvania $16802^{\circ}$

Y. Iga

Polytechnic University, Sagamihara, Japan ${ }^{f}$ 
G. D'Agostini, G. Marini, A. Nigro

Dipartimento di Fisica, Università 'La Sapienza' and INFN, Rome, Italy ${ }^{e}$

J.E. Cole, J.C. Hart

Rutherford Appleton Laboratory, Chilton, Didcot, Oxon, United Kingdom ${ }^{m}$

H. Abramowicz ${ }^{27}$, A. Gabareen, R. Ingbir, S. Kananov, A. Levy, O. Smith, A. Stern

Raymond and Beverly Sackler Faculty of Exact Sciences, School of Physics, Tel-Aviv University, Tel-Aviv, Israel ${ }^{d}$

M. Kuze, J. Maeda

Department of Physics, Tokyo Institute of Technology, Tokyo, Japan ${ }^{f}$

R. Hori, S. Kagawa ${ }^{28}$, N. Okazaki, S. Shimizu, T. Tawara

Department of Physics, University of Tokyo, Tokyo, Japan ${ }^{f}$

R. Hamatsu, H. Kaji ${ }^{29}$, S. Kitamura ${ }^{30}$, O. Ota, Y.D. Ri

Tokyo Metropolitan University, Department of Physics, Tokyo, Japan ${ }^{f}$

M.I. Ferrero, V. Monaco, R. Sacchi, A. Solano

Università di Torino and INFN, Torino, Italy ${ }^{e}$

M. Arneodo, M. Ruspa

Università del Piemonte Orientale, Novara, and INFN, Torino, Italy ${ }^{e}$

S. Fourletov, J.F. Martin

Department of Physics, University of Toronto, Toronto, Ontario, Canada M5S 1A7 a

S.K. Boutle ${ }^{18}$, J.M. Butterworth, C. Gwenlan ${ }^{31}$, T.W. Jones, J.H. Loizides, M.R. Sutton ${ }^{31}$, M. Wing

Physics and Astronomy Department, University College London, London, United Kingdom $^{m}$

B. Brzozowska, J. Ciborowski ${ }^{32}$, G. Grzelak, P. Kulinski, P. Łużniak ${ }^{33}$, J. Malka ${ }^{33}$, R.J. Nowak, J.M. Pawlak, T. Tymieniecka, A. Ukleja, A.F. Żarnecki

Warsaw University, Institute of Experimental Physics, Warsaw, Poland

M. Adamus, P. Plucinski ${ }^{34}$

Institute for Nuclear Studies, Warsaw, Poland

Y. Eisenberg, I. Giller, D. Hochman, U. Karshon, M. Rosin

Department of Particle Physics, Weizmann Institute, Rehovot, Israel ${ }^{c}$

E. Brownson, T. Danielson, A. Everett, D. Kçira, D.D. Reeder ${ }^{4}$, P. Ryan, A.A. Savin, W.H. Smith, H. Wolfe

Department of Physics, University of Wisconsin, Madison, Wisconsin 53706, USA ${ }^{n}$

S. Bhadra, C.D. Catterall, Y. Cui, G. Hartner, S. Menary, U. Noor, J. Standage, J. Whyte Department of Physics, York University, Ontario, Canada M3J 1P3 ${ }^{a}$ 
${ }^{1}$ supported by DESY, Germany

2 also affiliated with University College London, UK

${ }^{3}$ now at Humboldt University, Berlin, Germany

${ }^{4}$ retired

5 self-employed

${ }^{6}$ supported by Chonnam National University in 2005

7 supported by a scholarship of the World Laboratory Björn Wiik Research Project

8 supported by the research grant no. 1 P03B 04529 (2005-2008)

9 This work was supported in part by the Marie Curie Actions Transfer of Knowledge project COCOS (contract MTKD-CT-2004-517186)

${ }^{10}$ now at Univ. Libre de Bruxelles, Belgium

11 now at DESY group FEB, Hamburg, Germany

12 now at Stanford Linear Accelerator Center, Stanford, USA

13 now at University of Liverpool, UK

14 also at Institut of Theoretical and Experimental Physics, Moscow, Russia

15 also at INP, Cracow, Poland

16 on leave of absence from FPACS, AGH-UST, Cracow, Poland

17 partly supported by Moscow State University, Russia

18 also affiliated with DESY

19 now at CERN, Geneva, Switzerland

20 also at University of Tokyo, Japan

${ }^{21}$ now at Kobe University, Japan

${ }^{22}$ Ramón y Cajal Fellow

23 partly supported by Russian Foundation for Basic Research grant no. 05-02-39028NSFC-a

${ }^{24}$ EU Marie Curie Fellow

25 partially supported by Warsaw University, Poland

26 This material was based on work supported by the National Science Foundation, while working at the Foundation.

27 also at Max Planck Institute, Munich, Germany, Alexander von Humboldt Research Award

${ }^{28}$ now at KEK, Tsukuba, Japan

${ }^{29}$ now at Nagoya University, Japan

30 Department of Radiological Science

${ }^{31}$ PPARC Advanced fellow

32 also at Eódź University, Poland

33 Łódź University, Poland

34 supported by the Polish Ministry for Education and Science grant no. 1 P03B 14129

$\dagger$ deceased 
a supported by the Natural Sciences and Engineering Research Council of Canada (NSERC)

$b$ supported by the German Federal Ministry for Education and Research (BMBF), under contract numbers 05 HZ6PDA, 05 HZ6GUA, 05 HZ6VFA and 05 HZ4KHA

$c$ supported in part by the MINERVA Gesellschaft für Forschung GmbH, the Israel Science Foundation (grant no. 293/02-11.2) and the U.S.-Israel Binational Science Foundation

$d \quad$ supported by the German-Israeli Foundation and the Israel Science Foundation

$e$ supported by the Italian National Institute for Nuclear Physics (INFN)

$f$ supported by the Japanese Ministry of Education, Culture, Sports, Science and Technology (MEXT) and its grants for Scientific Research

$g$ supported by the Korean Ministry of Education and Korea Science and Engineering Foundation

$h$ supported by the Netherlands Foundation for Research on Matter (FOM)

$i$ supported by the Polish State Committee for Scientific Research, grant no. 620/E-77/SPB/DESY/P-03/DZ 117/2003-2005 and grant no. 1P03B07427/2004-2006

${ }^{j}$ partially supported by the German Federal Ministry for Education and Research $(\mathrm{BMBF})$

$k$ supported by RF Presidential grant N 8122.2006.2 for the leading scientific schools and by the Russian Ministry of Education and Science through its grant Research on High Energy Physics

$l$ supported by the Spanish Ministry of Education and Science through funds provided by CICYT

$m$ supported by the Particle Physics and Astronomy Research Council, UK

$n$ supported by the US Department of Energy

$o$ supported by the US National Science Foundation. Any opinion, findings and conclusions or recommendations expressed in this material are those of the authors and do not necessarily reflect the views of the National Science Foundation.

$p \quad$ supported by the Polish Ministry of Science and Higher Education as a scientific project (2006-2008)

$q$ supported by FNRS and its associated funds (IISN and FRIA) and by an Inter-University Attraction Poles Programme subsidised by the Belgian Federal Science Policy Office

$r$ supported by the Malaysian Ministry of Science, Technology and Innovation/Akademi Sains Malaysia grant SAGA 66-02-03-0048 


\section{Introduction}

Diffractive events in deep inelastic scattering (DIS) are characterised by the presence of a fast forward proton, a large rapidity gap (LRG) - an angular region between the scattered proton and the dissociated photon with no particle flow [1-6] - and a dissociated virtual photon $\gamma^{*}$. In recent years perturbative QCD (pQCD) has become a successful tool for describing diffractive events [4-7]. The cross section for diffractive DIS processes can be described by a convolution of universal diffractive parton distribution functions (dPDFs) and process-dependent coefficients, which can be calculated in pQCD [8]. At HERA, dPDFs have been determined using inclusive diffractive DIS data [4-6].

This paper presents measurements of dijet production in diffractive neutral current DIS with the ZEUS detector at HERA. The presence of a hard scale in such a process, either the virtuality of the photon or the large jet transverse momentum, is well suited for a pQCD analysis. Dijet processes are particularly sensitive to the density of gluons in the diffractive exchange (i.e. via $\gamma^{*} g \rightarrow q \bar{q}$, as shown in Fig. 1), and gluons have been shown to carry most of the momentum of the colourless exchange $[4,5,9]$. The measured differential cross sections are compared with leading-order (LO) and next-to-leading-order (NLO) QCD predictions using the available dPDFs. The results presented here benefit from higher statistics compared to previous measurements of the same process [10].

\section{Experimental set-up}

This analysis is based on $61 \mathrm{pb}^{-1}$ of data collected with the ZEUS detector at the HERA collider during the 1999-2000 data-taking period. During this period, HERA collided either electrons or positrons ${ }^{1}$ of $27.5 \mathrm{GeV}$ with protons of $920 \mathrm{GeV}$ at a centre-of-mass energy of $\sqrt{s}=318 \mathrm{GeV}$.

A detailed description of the ZEUS detector can be found elsewhere [11]. A brief outline of the components that are most relevant for this analysis is given below.

Charged particles are tracked in the central tracking detector (CTD) [12], which operates in a magnetic field of $1.43 \mathrm{~T}$ provided by a thin superconducting coil. The CTD consists of 72 cylindrical drift chamber layers, organised in 9 superlayers covering the polar-angle region $^{2} 15^{\circ}<\theta<164^{\circ}$. The transverse-momentum resolution for full-length tracks is

\footnotetext{
${ }^{1}$ In the following, for simplicity, the word positron will be used to denote both electrons and positrons. The integrated luminosity for $e^{-} p$ data is $3 \mathrm{pb}^{-1}$, while for $e^{+} p$ data is $58 \mathrm{pb}^{-1}$.

${ }^{2}$ The ZEUS coordinate system is a right-handed Cartesian system, with the $Z$ axis pointing in the proton beam direction, referred to as the "forward direction", and the $X$ axis pointing left towards the centre of HERA. The coordinate origin is at the nominal interaction point.
} 
$\sigma\left(p_{T}\right) / p_{T}=0.0058 p_{T} \oplus 0.0065 \oplus 0.0014 / p_{T}$, with $p_{T}$ in $\mathrm{GeV}$.

The high-resolution uranium-scintillator calorimeter (CAL) [13] consists of three parts: the forward (FCAL), the barrel (BCAL) and the rear (RCAL) calorimeters. Each part is subdivided transversely into towers and longitudinally into one electromagnetic section and either one (in RCAL) or two (in BCAL and FCAL) hadronic sections. The smallest subdivision of the calorimeter is called a cell. The CAL energy resolutions, as measured under test-beam conditions, are $\sigma(E) / E=0.18 / \sqrt{E}$ for electrons and $\sigma(E) / E=0.35 / \sqrt{E}$ for hadrons, with $E$ in $\mathrm{GeV}$.

During the 1999-2000 data-taking period, the forward plug calorimeter (FPC) [14], located in the beam hole of FCAL, extended the pseudorapidity coverage of the calorimeter up to values of $\eta \approx 5$. It consisted of a lead-scintillator calorimeter read out by wavelength shifters and photomultipliers.

In order to improve the detection of positrons scattered at low angles, the angular coverage in the rear direction was extended by means of the small rear tracking detector (SRTD) $[15,16]$. The SRTD consists of two planes of $1 \mathrm{~cm}$ wide and $0.5 \mathrm{~cm}$ thick scintillator strips glued on the front of RCAL. The orientations of the strips in the two planes are orthogonal. Scattered positrons were also detected in the rear hadron-electron separator (RHES) [17], a matrix of more than 10000 silicon diodes $400 \mu \mathrm{m}$ thick inserted in the RCAL.

The luminosity was measured using the bremsstrahlung process $e p \rightarrow e p \gamma$ with the luminosity monitor [18], a lead-scintillator calorimeter placed in the HERA tunnel at $Z=-107 \mathrm{~m}$.

\section{Kinematics}

Dijet production in diffractive DIS $\left(e p \rightarrow e+p+\mathrm{j} 1+\mathrm{j} 2+\mathrm{X}^{\prime}\right)$ is characterised by the simultaneous presence of a scattered positron, a scattered proton $p$ that escapes undetected down the beam pipe, and the photon-dissociative system $\mathrm{X}$, which contains the dijet system $\mathrm{j} 1+\mathrm{j} 2$, produced in the hard scattering along with the rest of the hadronic system $\mathrm{X}^{\prime}$ (see Fig. 1). Deep inelastic scattering of a positron on a proton is described by the following kinematic variables:

- $s=(P+k)^{2}$, the squared ep centre-of-mass energy, where $P$ and $k$ indicate the incoming proton and the incoming positron four-momenta, respectively;

- $Q^{2}=-q^{2}=-\left(k-k^{\prime}\right)^{2}$, the virtuality of $\gamma^{*}$, where $k^{\prime}$ is the four-momentum of the scattered positron;

- $W^{2}=(P+q)^{2}$, the centre-of-mass energy squared of the $\gamma^{*} p$ system. 
Diffractive events are further characterised by the variables:

- $M_{\mathrm{X}}$, the invariant mass of the photon-dissociative system;

- $t=\left(P-P^{\prime}\right)^{2}$, the squared four-momentum transfer at the proton vertex, where $P^{\prime}$ denotes the four-momentum of the scattered proton;

- $x_{\mathbb{P}}=\left(P-P^{\prime}\right) \cdot q / P \cdot q$, the momentum fraction lost by the proton;

- $\beta=Q^{2} / 2\left(P-P^{\prime}\right) \cdot q$, a measure of the fractional momentum of the diffractive exchange carried by the struck parton.

The description of the dijet system in the hadronic final state requires the use of additional variables:

- $z_{\mathbb{P}}$, the fraction of the momentum of the diffractive exchange carried by the parton participating in the hard process and defined as

$$
z_{\mathbb{P}}=\frac{q \cdot v}{q \cdot\left(P-P^{\prime}\right)}
$$

where $v$ is the four-momentum of the parton originating from the diffractive exchange;

- $x_{\gamma}$, the fractional momentum of the virtual photon participating in the hard process. In DIS, $x_{\gamma}$ is expected to be unity (direct photon). However, some models introduce the concept of a resolved virtual photon, where the $\gamma^{*}$ can fluctuate into a partonic state before participating in the hard interaction. For resolved photon processes, $x_{\gamma}$ is expected to be lower than unity. The variable $x_{\gamma}$ is defined as

$$
x_{\gamma}=\frac{P \cdot u}{P \cdot q},
$$

where $u$ is the four-momentum of the parton originating from the virtual photon.

\section{Theoretical models}

\subsection{QCD factorisation in diffraction}

The cross section for diffractive DIS processes at fixed $s$ depends in general on four independent variables, which are usually chosen to be $Q^{2}, \beta, x_{\mathbb{P}}$ and $t$. According to the QCD factorisation theorem [8], the cross section for inclusive diffraction, $\sigma\left(\gamma^{*} p \rightarrow \mathrm{X} p\right)$, can be written as

$$
\frac{d^{2} \sigma}{d x_{\mathbb{P}} d t}=\sum_{i=q, \bar{q}, g} \int d Q^{2} \int_{\beta}^{1} d \xi \hat{\sigma}^{\gamma^{*} i}\left(Q^{2}, \xi\right) f_{i}^{D}\left(x_{\mathbb{P}}, t, \xi, Q^{2}\right) .
$$


This expression is valid at fixed $x_{\mathbb{P}}$ and $t$ and for scales sufficiently large to permit the use of pQCD. The sum runs over all partons. The partonic cross-section $\hat{\sigma}^{\gamma^{*} i}\left(Q^{2}, \beta\right)$ for the hard subprocess involving the virtual photon and the parton $i$ is calculable in pQCD. The functions $f_{i}^{D}\left(x_{\mathbb{P}}, t, \beta, Q^{2}\right)$ are the dPDFs: they describe the probability to find in the proton a parton of kind $i$ carrying a fraction $x_{\mathbb{P}} \cdot \beta$ of its momentum with a probe of resolution $Q^{2}$ under the condition that the proton stays intact, with a momentum loss quantified by $x_{\mathbb{P}}$ and $t$. For diffractive production of dijets, Eq. (3) is rewritten as:

$$
\frac{d^{2} \sigma_{j j}}{d x_{\mathbb{P}} d t}=\sum_{i=q, \bar{q}, g} \int d Q^{2} \int_{z \mathbb{P}}^{1} d \xi \hat{\sigma}_{j j}^{\gamma^{*} i}\left(Q^{2}, \xi\right) f_{i}^{D}\left(x_{\mathbb{P}}, t, \xi, Q^{2}\right),
$$

where now $z_{\mathbb{P}}$ is the variable sensitive to the dPDFs and the subprocess cross section $\sigma^{\gamma^{*} i}$ is replaced by the cross section, $\sigma_{j j}^{\gamma^{*} i}$, for the reaction $\gamma^{*} i \rightarrow \mathrm{j} 1 \mathrm{j} 2$.

At HERA, the dPDFs have been determined within the QCD DGLAP formalism [19-22] by means of fits to inclusive diffractive DIS measurements with a procedure similar to that used to extract the standard proton PDFs from inclusive DIS data [23-28]. Consistency between the measured cross sections for semi-inclusive processes and calculations using these $\mathrm{dPDF}$ represents an experimental proof of the validity of the QCD factorization hypothesis in diffraction $[10,29]$.

Most of the dPDF parameterisations use Regge phenomenology arguments [30] to factorise the $\left(x_{\mathbb{P}}, t\right)$ from the $\left(\beta, Q^{2}\right)$ dependence. In the Regge approach, diffractive scattering proceeds via the exchange of the Pomeron trajectory. The dPDFs are then written as the product of the Pomeron flux (dependent on $x_{\mathbb{P}}$ and $t$ ) and parton distributions in the Pomeron (dependent on $\beta$ and $Q^{2}$ ). For $x_{\mathbb{P}}$ values substantially larger than 0.01 , the contribution of the subleading Reggeon trajectories may also have to be added.

\subsection{NLO calculation}

Predictions for diffractive dijet differential cross sections were calculated at order $\alpha_{S}^{2}$ with the program Disent [31] adapted for diffractive processes. The calculations were performed in the $\overline{M S}$ renormalisation scheme with five active flavours and the value of the strong coupling constant set to $\alpha_{s}\left(M_{Z}\right)=0.118$. The predictions were obtained with the renormalisation scale, $\mu_{\mathrm{R}}$, equal to $E_{\mathrm{T}, \mathrm{j} 1}^{*}$, where $E_{\mathrm{T}, \mathrm{j} 1}^{*}$ is the transverse energy of the highest transverse energy jet in the event (the leading jet) as measured in the $\gamma^{*} p$ centre-of-mass frame. The factorisation scale was set to $Q^{2}$.

The following dPDFs were used: 
- the ZEUS LPS+charm [6] - the result of an NLO DGLAP QCD fit to the inclusive diffractive structure functions measured by the ZEUS experiment with the leading proton spectrometer (LPS). In order to better constrain the dPDFs, measurements of $D^{*}$ production cross section in diffractive DIS [32] were also included. The fit was restricted to the region $x_{\mathbb{P}}<0.01$;

- the H1 2006 dPDFs [5] - the result of an NLO DGLAP QCD fit to a sample of inclusive diffractive structure functions measured by the H1 Collaboration. Two different parameterisations are available (Fit A and B) which differ in the gluon distribution. The fit was restricted to the region $Q^{2}>8.5 \mathrm{GeV}^{2}, z_{\mathbb{P}}<0.8$. Since the H1 measurements were not corrected for the contribution due to events where the proton dissociated into a low-mass state, in the comparison the calculations were renormalised by a factor $0.87[5]$;

- the Martin-Ryskin-Watt 2006 (MRW 2006) dPDFs [7] - the result of a fit to the same data set as for the H1 2006 fit. Regge factorisation is assumed only at the input scale. The dPDFs are then evolved with an inhomogeneous evolution equation analogous to that for the photon PDFs. The inhomogenous term accounts for the perturbative Pomeron-to-parton splitting.

The only theoretical source of uncertainty considered was that coming from the NLO calculations. This uncertainty was estimated by varying $\mu_{\mathrm{R}}$ by factors of 0.5 and 2 . Uncertainties of more than $20 \%$ were obtained. To compare with the data, the NLO predictions at the parton level were corrected to the hadron level using factors extracted from a MC program (see Section 5). The corrections were typically of the order of $10 \%$.

\section{Monte Carlo simulation}

Monte Carlo (MC) simulations were used to correct the data for acceptance and detector effects. Two different MC generators were used, RAPGAP [33] and SATRAP [34].

The RAPGAP MC is based on the factorised-Pomeron approach. The events were generated using the H1 fit 2 dPDFs [4]. No Reggeon contribution was included in this simulation. The parton-shower simulation is based on the MEPS [35] model. Resolved photon processes were also generated using RAPGAP with the GRV-G-HO [36] photon PDFs. Since the relative contributions of direct and resolved photon processes to the total cross section are a priori unknown, the RAPGAP direct and resolved samples were weighted in order to best describe the data. The RAPGAP MC was also used to extract the hadronisation corrections for the NLO calculation.

SATRAP is based on the Golec-Biernat-Wüsthoff (GBW) dipole model [34] and is interfaced to the RAPGAP framework. The parton-shower simulation in SATRAP is based on 
the Colour Dipole Model (CDM) [37]. This MC does not include the resolved-photon contribution to the $\gamma^{*} p$ cross section.

To estimate the inclusive DIS background, a sample of events was generated with DJANGOH [38].

All the above MC programs are interfaced to the HERACLES [39] event generator for the simulation of QED radiative processes and to JETSET [40] for the simulation of hadronisation according to the Lund model [41]. QED radiative corrections were typically between 5 and $10 \%$.

The ZEUS detector response was simulated with a program based on GEANT 3.13 [42]. The generated events were passed through the detector simulation, subjected to the same trigger requirements as the data, and processed by the same reconstruction and offline programs. The average of the acceptance-correction values obtained with RAPGAP and SATRAP was used to correct the data to the hadron level.

\section{Event reconstruction and data selection}

\subsection{DIS selection}

A three-level trigger system was used to select events online $[11,43]$. In the third-level trigger, a DIS positron candidate and energy deposition in the FPC lower than $20 \mathrm{GeV}$ were required. The scattered positron was identified both online and offline using a neuralnetwork algorithm [44]. The reconstruction of the scattered positron variables was carried out by combining the information from CAL, SRTD and HES. In order to select a DIS sample the following requirements were applied $[45,46]$ :

- the positron found in the RCAL had to lie outside a rectangular area of size $[-14,+12]$ $\mathrm{cm}$ in $X$ and $[-12,+12] \mathrm{cm}$ in $Y$, centred around the beam pipe. Further cuts on the fiducial area of the impact point of the positron on the RCAL surface were applied in order to exclude regions with significant inactive material [47];

- the energy of the scattered positron had to be greater than $10 \mathrm{GeV}$;

- the vertex of the event had to be in the range $\left|Z_{\mathrm{VTX}}\right|<50 \mathrm{~cm}$ to reject non-ep background.

The four-momentum of the hadronic final-state $\mathrm{X}$ was reconstructed using energy-flow objects (EFOs), which combine the information from the CAL and the CTD [48]. The EFOs were corrected for energy losses due to the inactive material present in the detector [49]. The variable $\delta=\sum_{i=e, \mathrm{EFO}}\left(E_{i}-p_{Z, i}\right)$, where the sum runs over the scattered positron 
and all the EFOs, was required to be $45<\delta<65 \mathrm{GeV}$. The variables $E_{i}$ and $p_{Z, i}$ denote the energy and the $Z$-component of the momentum of each term of the sum.

The $Q^{2}$ and $W$ variables were determined using the double-angle method [50]. Events were accepted if $5<Q^{2}<100 \mathrm{GeV}^{2}$ and $100<W<250 \mathrm{GeV}$.

\subsection{Jet selection}

The $k_{T}$-cluster algorithm in the longitudinal invariant mode [51] was applied to the corrected EFOs in the photon-proton centre-of-mass system $\left(\gamma^{*} p\right.$ frame) to reconstruct the jets. The jet variables in the $\gamma^{*} p$ frame are denoted by a star. After reconstructing the jets, the massless four-momenta were boosted to the laboratory frame where further energy corrections were determined and propagated back into the transverse energy of the jet, $E_{T, \text { jet }}^{*}$. Such corrections, obtained from a MC study, improved the correlation between hadron- and detector-level transverse energy of the jets [45]. The dijet sample was defined by requiring the events with at least two jets to fulfill the following constraints:

- $E_{T, \mathrm{j} 1}^{*}>5 \mathrm{GeV}$ and $E_{T, \mathrm{j} 2}^{*}>4 \mathrm{GeV}$, where the labels $\mathrm{j} 1$ and $\mathrm{j} 2$ refer to the jets with the highest and the second highest transverse energy, respectively;

- $-3.5<\eta_{\text {jet }}^{*}<0$, where $\eta_{\text {jet }}^{*}$ is the pseudorapidity of any of the jets;

- the pseudorapidity of the selected jets, boosted to the laboratory frame, had to lie in the range $\left|\eta_{\text {jet }}^{\mathrm{LAB}}\right|<2$.

\subsection{Diffractive selection}

Diffractive events are characterised by low values of $x_{\mathbb{P}}$ and by the presence of a LRG. The following selection criteria were applied $[45,46]$ :

- $E_{\mathrm{FPC}}<1 \mathrm{GeV}$, where $E_{\mathrm{FPC}}$ is the total energy in the FPC. The requirement of activity compatible with the noise level in the angular region covered by the FPC is equivalent to a rapidity-gap selection;

- $x_{\mathbb{P}}^{\mathrm{obs}}<0.03$ where $x_{\mathbb{P}}^{\mathrm{obs}}$ is the reconstructed value of $x_{\mathbb{P}}$ and is defined as:

$$
x_{\mathbb{P}}^{\mathrm{obs}}=\frac{Q^{2}+M_{\mathrm{X}}^{2}}{Q^{2}+W^{2}} .
$$

The mass of the diffractive system, $M_{\mathrm{X}}$, was reconstructed from the EFOs. The cut on $x_{\mathbb{P}}^{\text {obs }}$ reduces the contribution of Reggeon exchange and other non-diffractive background. 
After these cuts, the selected sample is still contaminated by diffractive events in which the $p$ dissociated into a low-mass system. This contamination was estimated by MC studies to be $f_{\text {pdiss }}=(16 \pm 4) \%$ [52] and was subtracted from the measurements independent of the kinematics.

The contamination of the non-diffractive background as a function of the applied diffractive selection cuts is shown in Fig. 2, through the distribution of $\eta_{\text {MAX }}$, where $\eta_{\text {MAX }}$ is the pseudorapidity in the laboratory frame of the most forward EFO with energy higher than $400 \mathrm{MeV}$, before and after applying cuts on the $E_{\mathrm{FPC}}$ and on $x_{\mathbb{P}}^{\text {obs }}$. The disagreement between the measured and the simulated distributions is the reason for not applying any requirement on $\eta_{\mathrm{MAX}}$, as was done in previous analyses $[9,32,53]$. After the $E_{\mathrm{FPC}}$ and $x_{\mathbb{P}}^{\mathrm{obs}}$ cuts, the non-diffractive background from DJANGOH was estimated to be $2.4 \%$ of the total selected events and neglected in further analysis. After all cuts, 5539 events remained.

\section{Systematic uncertainties}

The systematic uncertainties of the measured cross sections were calculated by varying the cuts and the analysis procedure. The systematic checks were the following:

- the energy measured by the CAL was varied by $\pm 3 \%$ in the MC to take into account the uncertainty on the CAL calibration, giving one of the largest uncertainties. Deviations from nominal cross section values were of the order of $\pm 5 \%$, but reached $\sim 15 \%$ in some bins;

- the energy scale of the scattered positron was varied in the MC by its uncertainty, $\pm 2 \%$. The resulting variation of the cross sections was always below $\pm 3 \%$;

- the position of the SRTD was changed in the MC by $\pm 2 \mathrm{~mm}$ in all directions to account for the uncertainty on its alignment. The change along the $Z$ direction gave the largest effect and in a few bins caused a cross section variation of $\pm 2 \%$;

- the model dependence of the acceptance corrections was estimated by using separately RAPGAP and SATRAP for unfolding the data. The variations from the central value (obtained using the average between RAPGAP and SATRAP) were typically of the order of $\pm 5 \%$ but reached $\sim \pm 10 \%$ in some bins.

The above systematic uncertainties, except those related to the energy scale of the calorimeter, were added in quadrature to determine the total systematic uncertainty. The uncertainties due to the energy scale and the proton dissociation subtraction $( \pm 4 \%)$ were added in quadrature and treated as correlated systematics. The energy scale uncertainty is quoted separately in the tables. 
The stability of the measurement was checked by varying the selection cuts as follows:

- the cut on the FPC energy was varied by $\pm 100 \mathrm{MeV}$ in the MC;

- the cut on the scattered-positron energy was lowered from 10 to $8 \mathrm{GeV}$;

- the fiducial region for the positron selection was enlarged and reduced by $0.5 \mathrm{~cm}$;

- the lower cut on $\delta$ was changed from 45 to $43 \mathrm{GeV}$.

The variations of the cross section induced by these stability checks were small, within $\pm 2 \%$, and were added in quadrature to the total systematic uncertainty. The uncertainty on the luminosity measurement $(2.25 \%)$ was not included.

The measurement was repeated with the addition of a cut on the value of $\eta_{\mathrm{MAX}}$. This estimates the uncertainty on the purity of the diffractive selection. A cut of $\eta_{\text {MAX }}<2.8$ was applied. The cross sections increased by $\sim 5 \%$ and the change was concentrated at high values of $x_{\mathbb{P}}^{\mathrm{obs}}$. No significant dependence on other variables was observed. This variation is listed in the tables for completeness but not included in the quoted uncertainties of the measurement.

\section{Results and discussion}

The single- and double-differential cross sections for the production of dijets in diffractive DIS have been measured for $5<Q^{2}<100 \mathrm{GeV}^{2}, 100<W<250 \mathrm{GeV}$ and $x_{\mathbb{P}}<0.03$, for jets in the pseudorapidity region $-3.5<\eta_{\mathrm{jet}}^{*}<0$, with $E_{T, \mathrm{j} 1}^{*}>5 \mathrm{GeV}$ and $E_{T, \mathrm{j} 2}^{*}>4 \mathrm{GeV}$. The cross sections refer to jets of hadrons and are corrected for QED effects.

The measured total cross section (given in Table 1) is:

$$
\sigma\left(e p \rightarrow e p+\mathrm{j} 1+\mathrm{j} 2+\mathrm{X}^{\prime}\right)=89.7 \pm 1.2(\text { stat }){ }_{-5.3}^{+3.2} \text { (syst. }{ }_{-3.7}^{+5.1} \text { (corr.) pb. }
$$

The values of the differential cross sections are averaged over the bin in which they are presented. For any variable $\kappa$, the cross section was determined as

$$
\frac{d \sigma}{d \kappa}=C \frac{N_{D}\left(1-f_{\text {pdiss }}\right)}{\mathcal{L} \Delta \kappa}
$$

where $N_{D}$ is the number of data events in a bin, $C$ includes the effects of the acceptance and the $\mathrm{QED}$ correction factor as determined from $\mathrm{MC}, \mathcal{L}$ is the integrated luminosity and $\Delta \kappa$ is the bin width.

The differential cross sections were measured as a function of $Q^{2}, W, x_{\mathbb{P}}^{\mathrm{obs}}, \beta, M_{\mathrm{X}}, E_{\mathrm{T}, \mathrm{J}}^{*}$, $\eta_{\mathrm{J}}^{*}, z_{\mathbb{P}}^{\mathrm{obs}}$ and $x_{\gamma}^{\mathrm{obs}}$. The variable $E_{\mathrm{T}, \mathrm{J}}^{*}\left(\eta_{\mathrm{J}}^{*}\right)$ stands for both $E_{\mathrm{T}, \mathrm{j} 1}^{*}\left(\eta_{\mathrm{j} 1}^{*}\right)$ and $E_{\mathrm{T}, \mathrm{j} 2}^{*}\left(\eta_{\mathrm{j} 2}^{*}\right)$ - in the 
corresponding cross section, it thus contributes two entries per event. The variable $z_{\mathbb{P}}^{\text {obs }}$ is an estimator of $z_{\mathbb{P}}$ and is calculated as

$$
z_{\mathbb{P}}^{\mathrm{obs}}=\frac{Q^{2}+M_{\mathrm{jj}}^{2}}{Q^{2}+M_{\mathrm{X}}^{2}}
$$

where $M_{\mathrm{jj}}$ is the invariant mass of the dijet system. The estimator of $x_{\gamma}, x_{\gamma}^{\mathrm{obs}}$, is

$$
x_{\gamma}^{\mathrm{obs}}=\frac{E_{\mathrm{T}, \mathrm{j} 1}^{\mathrm{LAB}} e^{-\eta_{\mathrm{j} 1}^{\mathrm{LAB}}}+E_{\mathrm{T}, \mathrm{j} 2}^{\mathrm{LAB}} e^{-\eta_{\mathrm{j} 2}^{\mathrm{LAB}}}}{\sum_{\text {hadr }}\left(E_{i}-p_{Z, i}\right)},
$$

where the sum in the denominator runs over all the hadrons. The values of the differential cross sections are presented in Tables 2-11 and shown in Figs. 3 and 4.

\subsection{Comparison to Monte Carlo models}

The RAPGAP and SATRAP MC programs are compared to the measured cross sections in Figs. 3 and 4. Since the MC predictions are not expected to describe the normalisation, the cross sections predicted by both MCs were normalised to the data. The total correlated uncertainty is shown as a shaded band in the figures. The comparison with MC predictions shows in general a reasonably good agreement with the shape of the data. The $E_{\mathrm{T}, \mathrm{J}}^{*}$ distribution is a steeply falling function as expected in pQCD (Fig. 4a) and the jets tend to populate the $\gamma^{*}$ fragmentation region.

The most prominent features of the data are the rise of the cross section with $x_{\mathbb{P}}^{\text {obs }}$, the peak at $z_{\mathbb{P}}^{\text {obs }} \sim 0.3$ and the tail of the cross section at low $x_{\gamma}^{\text {obs }}$ values. The requirement of two jets with high $E_{\mathrm{T}}$ suppresses the contribution of low values of $x_{\mathbb{P}}^{\text {obs }}$. The relatively low value of the peak position in the $z_{\mathbb{P}}^{\text {obs }}$ distribution indicates that in the majority of the events the dijet system is accompanied by additional hadronic activity. A disagreement between data and RAPGAP is observed at high $z_{\mathbb{P}}^{\text {obs }}$. In the high $z_{\mathbb{P}}^{\text {obs }}$ region, RAPGAP underestimates the number of events while SATRAP agrees with the data, possibly because of the presence of a mechanism for exclusive direct production. Most of the events are produced at large $x_{\gamma}^{\text {obs }}$ as expected in DIS. At low $x_{\gamma}^{\text {obs }}$, the description by RAPGAP is improved by the addition of the resolved photon contribution (16\%). 


\subsection{Comparison to NLO QCD predictions}

In Table 1, the four NLO predictions described in Section 4.2 are compared to the measured total cross section. The central values of the predictions using the H1 2006 - FitB and MRW 2006 dPDFs give the best description, while those using the H1 2006 - FitA and the ZEUS LPS+charm dPDFs are higher in normalisation.

The NLO predictions for the differential cross section are compared to the data in Figs. 5 and 6 . The estimated theoretical uncertainties are shown only for the calculations using the ZEUS LPS+charm dPDFs and are similar for all the other calculations. For ease of comparison the ratios of data to the ZEUS LPS+charm prediction are presented in Figs. 7 and 8. The variation due to the choice of the dPDFs is displayed with respect to the ZEUS LPS+charm in the same figure. In general the shape of the measured cross section is described by the NLO calculations within the theoretical uncertainties. However, only the predictions using the H1 2006 - FitB and MRW 2006 dPDFs are able to describe satisfactorily the data over the entire kinematic range.

The NLO predictions for the differential cross section are compared to the data in Figs. 9 and 10 , where the $z_{\mathbb{P}}^{\text {obs }}$ distribution is shown for different regions of $E_{\mathrm{T}, \mathrm{j} 1}^{*}$ and $Q^{2}$. Within the theoretical uncertainties, the H1 2006 - FitB and MRW 2006 dPDFs are compatible with the data. Since the major difference between the H1 2006 - FitB and Fit A is in the gluon dPDF, these data have a significant potential to further constrain the gluon dPDF.

\section{Conclusions}

The single- and double-differential cross sections for the production of dijets in diffractive DIS have been measured with the ZEUS detector in the kinematic region $5<Q^{2}<100$ $\mathrm{GeV}^{2}, 100<W<250 \mathrm{GeV}$ and $x_{\mathbb{P}}<0.03$, requiring at least two jets with $E_{T \text {,jet }}^{*}>4 \mathrm{GeV}$ in the pseudorapidity region $-3.5<\eta_{\mathrm{jet}}^{*}<0.0$ and the highest $E_{T}^{*}$ jet with $E_{T, \mathrm{j} 1}^{*}>5 \mathrm{GeV}$.

Two leading-logarithm parton-shower models, RAPGAP and SATRAP, describe the shape of the measured cross sections well. The measured cross sections are able to discriminate between NLO QCD calculations based on different dPDFs, showing a satisfactory agreement with the calculations using the H1 2006 - FitB and MRW 2006 dPDFs. This lends further support to the validity of QCD factorisation in hard diffractive scattering. Since the dPDFs used differ mostly in the gluon content, these data may have a significant potential to constrain the diffractive gluon distribution. 


\section{Acknowledgments}

We are grateful to the DESY Directorate for their strong support and encouragement. The effort of the HERA machine group is gratefully acknowledged. We thank the DESY computing and network services for their support. The design, construction and installation of the ZEUS detector have been made possible by the efforts of many people not listed as authors. We thank G. Watt and H. Jung for valuable discussions and suggestions. 


\section{References}

[1] ZEUS Coll., J. Breitweg et al., Eur. Phys. J. C 6, 43 (1999).

[2] ZEUS Coll., S. Chekanov et al., Eur. Phys. J. C 25, 169 (2002).

[3] ZEUS Coll, S. Chekanov et al., Nucl. Phys. B 713, 3 (2005).

[4] H1 Coll., C. Adloff et al., Z. Phys. C 76, 613 (1997).

[5] H1 Coll., A. Aktas et al., Eur. Phys. J. C 48, 715 (2006).

[6] ZEUS Coll., S. Chekanov et al., Eur. Phys. J. C 38, 43 (2004).

[7] A.D. Martin, M.G. Ryskin and G. Watt, Phys. Lett. B 644, 131 (2006).

[8] J.C. Collins, Phys. Rev. D 57, 3051 (1998);

J.C. Collins, J. Phys. G 28, 1069 (2002).

[9] ZEUS Coll., M. Derrick et al., Phys. Lett. B 356, 129 (1995).

[10] H1 Coll., A. Aktas et al., DESY-07-018, hep-ex/0703022.

[11] ZEUS Coll., U. Holm (ed.), The ZEUS Detector, Status Report (unpublished), DESY (1993), available on http://www-zeus.desy.de/bluebook/bluebook.html.

[12] N. Harnew et al., Nucl. Inst. Meth., A 279290 (1991);

B. Foster et al., Nucl. Phys. Proc. Suppl. B 32181 (1993);

B. Foster et al., Nucl. Inst. Meth. A 338, 254 (1994).

[13] M. Derrick et al., Nucl. Inst. Meth. A 309, 77 (1991);

A. Andresen et al., Nucl. Inst. Meth. A 309, 101 (1991);

A. Caldwell et al., Nucl. Inst. Meth. A 321, 356 (1992);

A. Bernstein et al., Nucl. Inst. Meth. A 336, 23 (1993).

[14] ZEUS Coll., A. Bamberger et al., FPC group, Nucl. Inst. Meth. A 450, 235 (2000).

[15] A. Bamberger et al., Nucl. Inst. Meth. A 401, 63 (1997).

[16] ZEUS Coll., S. Chekanov et al., Eur. Phys. J. C 21, 443 (2001).

[17] A. Dwurazny et al., Nucl. Inst. Meth. A 277, 176 (1989).

[18] J. Andruszków et al., Preprint DESY-92-066, DESY, 1992;

ZEUS Coll., M. Derrick et al., Z. Phys. C 63, 391 (1994);

J. Andruszków et al., Acta Phys. Pol. B 32, 2025 (2001).

[19] V.N. Gribov and L.N. Lipatov, Sov. J. Nucl. Phys. 15, 438 (1972).

[20] L.N. Lipatov, Sov. J. Nucl. Phys. 20, 94 (1975).

[21] G. Altarelli and G. Parisi, Nucl. Phys. B 126, 298 (1977). 
[22] Yu.L. Dokshitzer, Sov. Phys. JETP 46, 641 (1977).

[23] A.D. Martin et al., Eur. Phys. J. C 23, 73 (2002).

[24] A.D. Martin et al., Eur. Phys. J. C 28, 455 (2002).

[25] J. Pumplin et al., JHEP 0207, 012 (2002).

[26] H1 Coll., C. Adloff et al., Eur. Phys. J. C 30, 1 (2003).

[27] ZEUS Coll., S. Chekanov et al., Phys. Rev. D 67, 012007 (2003).

[28] ZEUS Coll., S. Chekanov et al., Eur. Phys. J. C 42, 1 (2005).

[29] H1 Coll., A. Aktas et al., DESY-06-164, hep-ex/0610076.

[30] P.D.B. Collins, An Introduction to Regge Theory and High-Energy Physics, Cambridge University Press, Cambridge, (1977);

A.C. Irving and R.P. Worden, Phys. Rept. 34, 117 (1977);

A.B. Kaidalov, Regge Poles in QCD, "At the Frontier of Particle Physics: Handbook of QCD" , M. Shifman (ed.), World Scientific (2002) (also preprint hep-ph/0103011).

[31] S. Catani and M.H. Seymour, Nucl. Phys. B 485, 291 (1997);

Erratum-ibid. B 510503 (1997).

[32] ZEUS Coll., S. Chekanov et al., Phys. Rev. D 69, 012004 (2004).

[33] H. Jung, The RAPGAP Monte Carlo for Deep Inelastic Scattering version 2.08/00 (2001). See http://www.desy.de/ jung/rapgap/.

[34] K. Golec-Biernat and W. Wüsthoff, Phys. Rev. D 59, (1999);

K. Golec-Biernat and W. Wüsthoff, Phys. Rev. D 60, (1999);

K. Golec-Biernat and W. Wüsthoff, Eur. Phys. J. C 20, 313 (2001).

[35] G. Ingelman, A. Edin and J. Rathsman, Comp. Phys. Comm. 101, 108 (1997).

[36] M. Glück, E. Reya and A. Vogt, Phys. Rev. D 45, 3986 (1992);

M. Glück, E. Reya and A. Vogt, Phys. Rev. D 46, 1973 (1992).

[37] G. Gustafson and U. Pettersson, Nucl. Phys. B 306, 746 (1988).

[38] K. Charchula, G.A. Schuler and H. Spiesberger, Comp. Phys. Comm. 81, 381 (1994).

[39] A. Kwiatowski, H. Spiesberger and H.-J. Möhring, Comp. Phys. Comm. 69, 155 (1992).

[40] M. Bengtsson and T. Sjöstrand, Comp. Phys. Comm. 46, 43 (1987);

T. Sjöstrand, Comp. Phys. Comm. 82, 74 (1994).

[41] B. Andersson et al., Phys. Rep. 97, 31 (1983).

[42] R. Brun et al., preprint CERN-DD/EE/84-1, CERN, 1987. 
[43] W.H. Smith, K. Tokushuku and L.W. Wiggers, Proc. Computing in High Energy Physics (CHEP), Annecy, France, Sept. 1992, C. Verkerk and W. Wojcik (eds.), p. 222. CERN, Geneva, Switzerland (1992). Also in preprint DESY 92-150B.

[44] H. Abramowicz, A. Caldwell and R. Sinkus, Nucl. Inst. Meth. A 365, 508 (1995);

R. Sinkus and T. Voss, Nucl. Inst. Meth. A 391, 360 (1997).

[45] A. Bonato, Ph. D. Thesis, University of Hamburg, (2007) (in preparation).

[46] T. Tawara, Ph. D. Thesis, University of Tokyo, (2007) (unpublished).

[47] T. Yamashita, Ph. D. Thesis, University of Tokyo, (2001) (unpublished).

[48] G.M. Briskin, Ph. D. Thesis, Tel Aviv University, (1998) (unpublished).

[49] M. Turcato, Ph. D. Thesis, Padova University, (2003) (unpublished).

[50] S. Bentvelsen, J. Engelen and P. Kooijman, Proc. Workshop on Physics at HERA, W. Buchmuller and G. Ingelman (ed.), Vol.1, p.23, DESY, Hamburg, Germany (1992).

[51] S. Catani et al., Nucl. Phys. B 406, 187 (1993).

[52] ZEUS Coll., S. Chekanov et al., Nucl. Phys. B 672, 3 (2003).

[53] ZEUS Coll., S. Chekanov et al., Eur. Phys. J. C 51, 301 (2007). 


\begin{tabular}{|c|c|c|c|c|c|c|}
\hline & $\begin{array}{c}\sigma \\
(\mathrm{pb})\end{array}$ & $\begin{array}{c}\delta_{\text {stat }} \\
(\mathrm{pb})\end{array}$ & $\begin{array}{c}\delta_{\text {syst }} \\
(\mathrm{pb})\end{array}$ & $\begin{array}{c}\delta_{\mathrm{ES}} \\
(\mathrm{pb})\end{array}$ & $\begin{array}{c}\delta_{\text {theor }} \\
(\mathrm{pb})\end{array}$ & $\begin{array}{c}\Delta_{\text {DIFFR }} \\
(\mathrm{pb})\end{array}$ \\
\hline \hline Data & 89.7 & 1.2 & ${ }_{-5.3}^{+3.2}$ & ${ }_{-3.7}^{+5.1}$ & - & +4.0 \\
ZEUS LPS+charm & 120.3 & - & - & - & ${ }_{-18.3}^{+29.4}$ & - \\
H1 2006 - Fit A & 130.2 & - & - & - & ${ }_{-19.9}^{+31.2}$ & - \\
H1 2006 - Fit B & 102.5 & - & - & - & ${ }_{-15.6}^{+24.7}$ & - \\
MRW 2006 & 99.3 & - & - & - & ${ }_{-14.7}^{+23.4}$ & - \\
\hline \hline
\end{tabular}

Table 1: Total cross section for the production of diffractive dijets compared to expectations of NLO calculations using various $d P D F s$ as indicated in the Table. The cross section is given for jets with $E_{T, \mathrm{j} 1}^{*}>5 \mathrm{GeV}, E_{T, \mathrm{j} 2}^{*}>4 \mathrm{GeV},-3.5<\eta_{\mathrm{jet}}^{*}<0$ and in the range of $5<Q^{2}<100 \mathrm{GeV}^{2}, 100<W<250 \mathrm{GeV}$ and $x_{\mathbb{P}}<0.03$. The statistical, $\delta_{\mathrm{stat}}$, uncorrelated systematic, $\delta_{\mathrm{syst}}$, and energy scale uncertainties, $\delta_{\mathrm{ES}}$, are quoted separately. The theoretical uncertainty on the NLO calculations, $\delta_{\text {theor }}$, is quoted in the sixth column. The difference with the measured cross section with and without $\eta_{\mathrm{MAX}}$ cut, $\Delta_{\mathrm{DIFFR}}$, is presented in the last column. The uncertainties on the proton dissociation subtraction and the luminosity measurement are not presented in the table. 


\begin{tabular}{|l|l|l|l|l|l|}
\hline $\begin{array}{c}Q^{2} \text { bin } \\
\left(\mathrm{GeV}^{2}\right)\end{array}$ & $\begin{array}{c}d \sigma / d Q^{2} \\
\left(\mathrm{pb} / \mathrm{GeV}^{2}\right)\end{array}$ & $\begin{array}{c}\delta_{\text {stat }} \\
\left(\mathrm{pb} / \mathrm{GeV}^{2}\right)\end{array}$ & $\begin{array}{l}\delta_{\text {syst }} \\
\left(\mathrm{pb} / \mathrm{GeV}^{2}\right)\end{array}$ & $\begin{array}{l}\delta_{\mathrm{ES}} \\
\left(\mathrm{pb} / \mathrm{GeV}^{2}\right)\end{array}$ & $\begin{array}{c}\Delta_{\text {DIFFR }} \\
\left(\mathrm{pb} / \mathrm{GeV}^{2}\right)\end{array}$ \\
\hline \hline 5,8 & 7.4 & \pm 0.3 & ${ }_{-0.5}^{+0.3}$ & ${ }_{-0.5}^{+0.5}$ & 0.1 \\
\hline 8,12 & 4.2 & \pm 0.2 & ${ }_{-0.3}^{+0.2}$ & ${ }_{-0.3}^{+0.3}$ & 0.1 \\
\hline 12,17 & 2.6 & \pm 0.1 & ${ }_{-0.2}^{+0.2}$ & ${ }_{-0.2}^{+0.2}$ & 0.2 \\
\hline 17,25 & 1.38 & \pm 0.06 & ${ }_{-0.13}^{+0.09}$ & ${ }_{-0.08}^{+0.08}$ & 0.06 \\
\hline 25,35 & 0.94 & \pm 0.04 & ${ }_{-0.07}^{+0.07}$ & ${ }_{-0.05}^{+0.06}$ & 0.06 \\
\hline 35,50 & 0.53 & \pm 0.03 & ${ }_{-0.03}^{+0.02}$ & ${ }_{-0.03}^{+0.03}$ & 0.01 \\
\hline 50,70 & 0.27 & \pm 0.02 & ${ }_{-0.01}^{+0.02}$ & ${ }_{-0.01}^{+0.01}$ & 0.02 \\
\hline 70,100 & 0.116 & \pm 0.008 & ${ }_{-0.003}^{+0.018}$ & ${ }_{-0.005}^{+0.005}$ & 0.018 \\
\hline \hline
\end{tabular}

Table 2: $\quad$ Values of the differential cross section as a function of $Q^{2}$ for the production of diffractive dijets. The range over which the cross section is averaged is given in the first column. Other details as in the caption of Table 1.

\begin{tabular}{|c|l|l|l|l|l|}
\hline $\begin{array}{c}W \text { bin } \\
(\mathrm{GeV})\end{array}$ & $\begin{array}{c}d \sigma / d W \\
(\mathrm{pb} / \mathrm{GeV})\end{array}$ & $\begin{array}{c}\delta_{\text {stat }} \\
(\mathrm{pb} / \mathrm{GeV})\end{array}$ & $\begin{array}{l}\delta_{\text {syst }} \\
(\mathrm{pb} / \mathrm{GeV})\end{array}$ & $\begin{array}{l}\delta_{\mathrm{ES}} \\
(\mathrm{pb} / \mathrm{GeV})\end{array}$ & $\begin{array}{c}\Delta_{\text {DIFFR }} \\
(\mathrm{pb} / \mathrm{GeV})\end{array}$ \\
\hline \hline 100,125 & 0.26 & \pm 0.01 & ${ }_{-0.03}^{+0.03}$ & ${ }_{-0.01}^{+0.01}$ & 0.01 \\
\hline 125,150 & 0.41 & \pm 0.02 & ${ }_{-0.03}^{+0.04}$ & ${ }_{-0.03}^{+0.02}$ & 0.03 \\
\hline 150,175 & 0.67 & \pm 0.03 & ${ }_{-0.06}^{+0.04}$ & ${ }_{-0.04}^{+0.04}$ & 0.03 \\
\hline 175,200 & 0.68 & \pm 0.02 & ${ }_{-0.04}^{+0.03}$ & ${ }_{-0.04}^{+0.05}$ & 0.01 \\
\hline 200,225 & 0.77 & \pm 0.03 & ${ }_{-0.03}^{+0.06}$ & ${ }_{-0.05}^{+0.05}$ & 0.05 \\
\hline 225,250 & 0.82 & \pm 0.03 & ${ }_{-0.06}^{+0.03}$ & ${ }_{-0.05}^{+0.05}$ & 0.02 \\
\hline \hline
\end{tabular}

Table 3: Values of the differential cross section as a function of $W$. Other details as in the caption of Table 2. 


\begin{tabular}{|c|l|l|l|l|l|}
\hline $\begin{array}{c}M_{X} \text { bin } \\
(\mathrm{GeV})\end{array}$ & $\begin{array}{c}d \sigma / d M_{X} \\
(\mathrm{pb} / \mathrm{GeV})\end{array}$ & $\begin{array}{c}\delta_{\text {stat }} \\
(\mathrm{pb} / \mathrm{GeV})\end{array}$ & $\begin{array}{l}\delta_{\text {syst }} \\
(\mathrm{pb} / \mathrm{GeV})\end{array}$ & $\begin{array}{l}\delta_{\mathrm{ES}} \\
(\mathrm{pb} / \mathrm{GeV})\end{array}$ & $\begin{array}{c}\Delta_{\text {DIFFR }} \\
(\mathrm{pb} / \mathrm{GeV})\end{array}$ \\
\hline \hline 9,14 & 1.31 & \pm 0.07 & ${ }_{-0.08}^{+0.02}$ & ${ }_{-0.06}^{+0.05}$ & -0.03 \\
\hline 14,20 & 4.3 & \pm 0.1 & ${ }_{-0.2}^{+0.2}$ & ${ }_{-0.2}^{+0.2}$ & 0.1 \\
\hline 20,26 & 4.5 & \pm 0.1 & ${ }_{-0.4}^{+0.2}$ & ${ }_{-0.2}^{+0.2}$ & 0.0 \\
\hline 26,32 & 3.1 & \pm 0.1 & ${ }_{-0.3}^{+0.2}$ & ${ }_{-0.2}^{+0.3}$ & -0.1 \\
\hline 32,42 & 1.13 & \pm 0.05 & ${ }_{-0.06}^{+0.08}$ & ${ }_{-0.09}^{+0.12}$ & 0.07 \\
\hline \hline
\end{tabular}

Table 4: Values of the differential cross sections with respect to $M_{X}$. Other details as in the caption of Table 2.

\begin{tabular}{|c|c|c|c|c|c|c|}
\hline $\begin{array}{c}\beta \text { bin } \\
\left(\times 10^{-2}\right)\end{array}$ & $\begin{array}{c}d \sigma / d \beta \\
(\mathrm{pb})\end{array}$ & & $\begin{array}{l}\delta_{\text {stat }} \\
(\mathrm{pb})\end{array}$ & $\begin{array}{l}\delta_{\text {syst }} \\
(\mathrm{pb})\end{array}$ & $\begin{array}{l}\delta_{\mathrm{ES}} \\
(\mathrm{pb})\end{array}$ & $\begin{array}{c}\Delta_{\text {DIFFR }} \\
(\mathrm{pb})\end{array}$ \\
\hline $0.32,0.63$ & 1220 & \pm & 102 & $\begin{array}{l}+30 \\
-75\end{array}$ & $\begin{array}{l}+148 \\
{ }_{-135}\end{array}$ & -69 \\
\hline $0.63,1.26$ & 2124 & \pm & 94 & $\begin{array}{l}+153 \\
{ }_{-221}\end{array}$ & $\begin{array}{l}+196 \\
{ }_{-177}^{+196}\end{array}$ & -11 \\
\hline $1.26,2.51$ & 1736 & \pm & 62 & $\begin{array}{l}+108 \\
-133\end{array}$ & $\begin{array}{l}+112 \\
{ }_{-109}\end{array}$ & 46 \\
\hline $2.51,5.01$ & 923 & \pm & 32 & $\begin{array}{l}+40 \\
-83 \\
\end{array}$ & $\begin{array}{l}+55 \\
-50 \\
\end{array}$ & 3 \\
\hline $5.01,10.00$ & 324 & \pm & 12 & $\begin{array}{l}+9 \\
-18 \\
\end{array}$ & $\begin{array}{l}+14 \\
-17\end{array}$ & 3 \\
\hline $10.00,19.95$ & 81.8 & \pm & 3.8 & $\begin{array}{l}+4.3 \\
-2.7\end{array}$ & $\begin{array}{l}+3.5 \\
-4.1 \\
\end{array}$ & 4.1 \\
\hline $19.95,39.81$ & 9.7 & \pm & 0.8 & $\begin{array}{l}+0.5 \\
-0.5 \\
\end{array}$ & $\begin{array}{l}+0.5 \\
-0.6 \\
\end{array}$ & 0.4 \\
\hline
\end{tabular}

Table 5: Values of the differential cross sections with respect to $\beta$. Other details as in the caption of Table 2. 


\begin{tabular}{|c|c|c|c|c|c|}
\hline $\begin{array}{l}x_{\mathbb{P}}^{\text {obs }} \text { bin } \\
\left(\times 10^{-2}\right)\end{array}$ & $\begin{array}{c}x_{\mathbb{P}}^{\mathrm{obs}} d \sigma / d x_{\mathbb{P}}^{\mathrm{obs}} \\
(\mathrm{pb})\end{array}$ & $\begin{array}{l}\delta_{\text {stat }} \\
(\mathrm{pb})\end{array}$ & $\begin{array}{l}\delta_{\text {syst }} \\
(\mathrm{pb})\end{array}$ & $\begin{array}{l}\delta_{\mathrm{ES}} \\
(\mathrm{pb})\end{array}$ & $\begin{array}{c}\Delta_{\text {DIFFR }} \\
(\mathrm{pb})\end{array}$ \\
\hline $0.25,0.50$ & 24.3 & $\pm \quad 1.8$ & $\begin{array}{l}+0.8 \\
-1.5 \\
\end{array}$ & $\begin{array}{l}+1.0 \\
{ }_{-1.1}\end{array}$ & -0.5 \\
\hline $0.50,0.79$ & 93 & $\pm \quad 5$ & $\begin{array}{l}+1 \\
-1\end{array}$ & $\begin{array}{l}+4 \\
-5\end{array}$ & 0 \\
\hline $0.79,1.26$ & 195 & $\pm \quad 7$ & $\begin{array}{l}+3 \\
{ }_{-9} \\
\end{array}$ & $\begin{array}{l}+9 \\
-10 \\
\end{array}$ & 2 \\
\hline $1.26,1.99$ & 306 & \pm 10 & $\begin{array}{l}+10 \\
-25 \\
\end{array}$ & $\begin{array}{l}+17 \\
-17 \\
\end{array}$ & 1 \\
\hline $1.99,3.00$ & 409 & \pm 13 & $\begin{array}{l}+33 \\
-33 \\
\end{array}$ & $\begin{array}{l}+35 \\
-30 \\
\end{array}$ & 26 \\
\hline
\end{tabular}

Table 6: Values of the differential cross sections with respect to $x_{\mathbb{P}}^{\text {obs }}$. Other details as in the caption of Table 2.

\begin{tabular}{|c|l|l|l|l|l|}
\hline $\begin{array}{c}E_{\mathrm{T}, \mathrm{J}}^{*} \text { bin } \\
(\mathrm{GeV})\end{array}$ & $\begin{array}{c}d \sigma / d E_{\mathrm{T}, \mathrm{J}}^{*} \\
(\mathrm{pb} / \mathrm{GeV})\end{array}$ & $\begin{array}{c}\delta_{\text {stat }} \\
(\mathrm{pb} / \mathrm{GeV})\end{array}$ & $\begin{array}{l}\delta_{\text {syst }} \\
(\mathrm{pb} / \mathrm{GeV})\end{array}$ & $\begin{array}{l}\delta_{\mathrm{ES}} \\
(\mathrm{pb} / \mathrm{GeV})\end{array}$ & $\begin{array}{c}\Delta_{\text {DIFFR }} \\
(\mathrm{pb} / \mathrm{GeV})\end{array}$ \\
\hline \hline $4,5.5$ & 51.7 & \pm 1.4 & ${ }_{-3.7}^{+3.3}$ & ${ }_{-2.9}^{+2.6}$ & 2.9 \\
\hline $5.5,7.5$ & 39.8 & \pm 1.1 & ${ }_{-2.8}^{+2.6}$ & ${ }_{-2.0}^{+2.2}$ & 1.8 \\
\hline $7.5,9.5$ & 9.7 & \pm 0.3 & ${ }_{-0.9}^{+0.7}$ & ${ }_{-0.9}^{+0.8}$ & 0.2 \\
\hline $9.5,11.5$ & 2.3 & \pm 0.1 & ${ }_{-0.1}^{+0.1}$ & ${ }_{-0.2}^{+0.3}$ & 0.1 \\
\hline $11.5,13.5$ & 0.65 & \pm 0.06 & ${ }_{-0.01}^{+0.03}$ & ${ }_{-0.11}^{+0.08}$ & 0.03 \\
\hline $13.5,16$ & 0.11 & \pm 0.02 & ${ }_{-0.02}^{+0.02}$ & ${ }_{-0.03}^{+0.01}$ & 0.00 \\
\hline \hline
\end{tabular}

Table 7: Values of the differential cross sections with respect to $E_{\mathrm{T}, \mathrm{J}}^{*}$. Other details as in the caption of Table 2. 


\begin{tabular}{|c|l|c|l|l|l|}
\hline$\eta_{\mathrm{J}}^{*}$ bin & $\begin{array}{c}d \sigma / d \eta_{\mathrm{J}}^{*} \\
(\mathrm{pb})\end{array}$ & $\begin{array}{c}\delta_{\text {stat }} \\
(\mathrm{pb})\end{array}$ & $\begin{array}{l}\delta_{\text {syst }} \\
(\mathrm{pb})\end{array}$ & $\begin{array}{l}\delta_{\mathrm{ES}} \\
(\mathrm{pb})\end{array}$ & $\begin{array}{c}\Delta_{\text {DIFFR }} \\
(\mathrm{pb})\end{array}$ \\
\hline \hline$-3.5,-3.0$ & 56.6 & \pm 1.9 & ${ }_{-3.8}^{+2.5}$ & ${ }_{-7.6}^{+7.8}$ & 1.6 \\
\hline$-3.0,-2.5$ & 98.8 & \pm 2.9 & ${ }_{-6.2}^{+3.6}$ & ${ }_{-7.1}^{+7.2}$ & 1.8 \\
\hline$-2.5,-2.0$ & 89.6 & \pm 2.6 & ${ }_{-6.0}^{+5.7}$ & ${ }_{-4.9}^{+5.1}$ & 4.8 \\
\hline$-2.0,-1.5$ & 66.1 & \pm 2.1 & ${ }_{-4.2}^{+4.1}$ & ${ }_{-4.1}^{+3.7}$ & 3.4 \\
\hline$-1.5,-1.0$ & 35.2 & \pm 1.3 & ${ }_{-2.6}^{+3.3}$ & ${ }_{-2.0}^{+2.7}$ & 3.0 \\
\hline$-1.0,-0.5$ & 13.2 & \pm 0.7 & ${ }_{-1.3}^{+1.4}$ & ${ }_{-1.3}^{+1.3}$ & 1.1 \\
\hline$-0.5,0.0$ & 2.1 & \pm 0.2 & ${ }_{-0.5}^{+0.4}$ & ${ }_{-0.3}^{+0.4}$ & -0.2 \\
\hline \hline
\end{tabular}

Table 8: Values of the differential cross sections with respect to $\eta_{\mathrm{J}}^{*}$. Other details as in the caption of Table 2.

\begin{tabular}{|c|c|c|l|l|l|}
\hline$z_{\mathbb{P}}^{\text {obs }}$ & $\begin{array}{c}d \sigma / d z_{\mathbb{P}}^{\text {obs }} \\
(\mathrm{pb})\end{array}$ & $\begin{array}{c}\delta_{\text {stat }} \\
(\mathrm{pb})\end{array}$ & $\begin{array}{l}\delta_{\text {syst }} \\
(\mathrm{pb})\end{array}$ & $\begin{array}{l}\delta_{\mathrm{ES}} \\
(\mathrm{pb})\end{array}$ & $\begin{array}{c}\Delta_{\text {DIFFR }} \\
(\mathrm{pb})\end{array}$ \\
\hline \hline $0,0.125$ & 24.5 & \pm 2.0 & ${ }_{-2.3}^{+0.9}$ & ${ }_{-2.0}^{+3.5}$ & -1.9 \\
\hline $0.125,0.25$ & 134.6 & \pm 5.4 & ${ }_{-13.8}^{+8.8}$ & ${ }_{-10.8}^{+12.3}$ & 0.7 \\
\hline $0.25,0.375$ & 155.1 & \pm 5.7 & ${ }_{-12.5}^{+9.6}$ & ${ }_{-9.8}^{+10.1}$ & 5.2 \\
\hline $0.375,0.5$ & 133.7 & \pm 5.1 & ${ }_{-10.2}^{+8.3}$ & ${ }_{-8.1}^{+6.1}$ & 5.7 \\
\hline $0.5,0.625$ & 100.6 & \pm 4.2 & ${ }_{-7.8}^{+5.8}$ & ${ }_{-5.4}^{+5.2}$ & 2.4 \\
\hline $0.625,0.75$ & 80.4 & \pm 3.6 & ${ }_{-2.8}^{+1.3}$ & ${ }_{-3.8}^{+3.8}$ & 0.5 \\
\hline $0.75,0.875$ & 55.5 & \pm 2.8 & ${ }_{-3.1}^{+1.7}$ & ${ }_{-2.8}^{+2.8}$ & -1.5 \\
\hline $0.875,1$ & 31.5 & \pm 2.1 & ${ }_{-4.0}^{+3.1}$ & ${ }_{-1.5}^{+2.2}$ & -1.3 \\
\hline \hline
\end{tabular}

Table 9: Values of the differential cross sections with respect to $z_{\mathbb{P}}^{\text {obs }}$. Other details as in the caption of Table 2. 


\begin{tabular}{|c|c|c|l|l|l|}
\hline$x_{\gamma}^{\text {obs }}$ bin & $\begin{array}{c}d \sigma / d x_{\gamma}^{\text {obs }} \\
(\mathrm{pb})\end{array}$ & $\begin{array}{c}\delta_{\text {stat }} \\
(\mathrm{pb})\end{array}$ & $\begin{array}{l}\delta_{\text {syst }} \\
(\mathrm{pb})\end{array}$ & $\begin{array}{l}\delta_{\mathrm{ES}} \\
(\mathrm{pb})\end{array}$ & $\begin{array}{c}\Delta_{\text {DIFFR }} \\
(\mathrm{pb})\end{array}$ \\
\hline \hline $0,0.25$ & 5.3 & \pm 0.6 & ${ }_{-0.5}^{+0.8}$ & ${ }_{-0.2}^{+0.6}$ & 0.7 \\
\hline $0.25,0.5$ & 25.0 & \pm 1.3 & ${ }_{-1.5}^{+2.8}$ & ${ }_{-2.2}^{+1.5}$ & 2.6 \\
\hline $0.5,0.75$ & 87.4 & \pm 3.1 & ${ }_{-5.7}^{+4.5}$ & ${ }_{-8.2}^{+7.8}$ & 3.5 \\
\hline $0.75,1$ & 240.5 & \pm 6.7 & ${ }_{-17.2}^{+11.0}$ & ${ }_{-11.7}^{+12.4}$ & 5.9 \\
\hline \hline
\end{tabular}

Table 10: Values of the differential cross sections with respect to $x_{\gamma}^{\text {obs }}$. Other details as in the caption of Table 2. 


\begin{tabular}{|c|c|c|c|c|c|}
\hline$z_{\mathbb{P}}^{\mathrm{obs}}$ bin & $\begin{array}{c}d \sigma / d z_{\mathbb{P}}^{\mathrm{obs}} d E_{T, j 1}^{*} \\
(\mathrm{pb} / \mathrm{GeV})\end{array}$ & $\begin{array}{c}\delta_{\text {stat }} \\
(\mathrm{pb} / \mathrm{GeV})\end{array}$ & $\begin{array}{l}\delta_{\text {syst }} \\
(\mathrm{pb} / \mathrm{GeV})\end{array}$ & $\begin{array}{l}\delta_{\mathrm{ES}} \\
(\mathrm{pb} / \mathrm{GeV})\end{array}$ & $\begin{array}{c}\Delta_{\text {DIFFR }} \\
(\mathrm{pb} / \mathrm{GeV})\end{array}$ \\
\hline \multicolumn{6}{|c|}{$5.0<E_{T, j 1}^{*}<6.5 \mathrm{GeV}\left(<E_{T, j 1}^{*}>=5.7 \mathrm{GeV}\right)$} \\
\hline $0,0.25$ & 31.9 & \pm 1.5 & $\begin{array}{l}+2.1 \\
-4.2\end{array}$ & $\begin{array}{l}+1.9 \\
{ }_{-1.4}^{+1.9}\end{array}$ & -1.2 \\
\hline $0.25,0.375$ & 53.1 & $\pm \quad 2.6$ & $\begin{array}{ll}+4.2 \\
-4.6 \\
\end{array}$ & $\begin{array}{l}+2.3 \\
-2.3 \\
\end{array}$ & 3.0 \\
\hline $0.375,0.5$ & 46.7 & \pm 2.4 & $\begin{array}{l}+3.0 \\
-3.0\end{array}$ & $\begin{array}{l}+1.9 \\
-2.4\end{array}$ & 2.5 \\
\hline $0.5,0.625$ & 35.3 & \pm 2.1 & $\begin{array}{l}+1.1 \\
-1.7\end{array}$ & $\begin{array}{l}+1.6 \\
{ }_{-1.8}^{+}\end{array}$ & 1.0 \\
\hline $0.625,0.75$ & 29.3 & $\pm \quad 1.9$ & $\begin{array}{l}+0.3 \\
-1.9 \\
\end{array}$ & $\begin{array}{l}+1.3 \\
-1.2 \\
\end{array}$ & -0.8 \\
\hline $0.75,0.875$ & 18.4 & $\pm \quad 1.4$ & $\begin{array}{l}+1.2 \\
-1.9\end{array}$ & $\begin{array}{l}+0.8 \\
-0.8\end{array}$ & -1.0 \\
\hline $0.875,1$ & 11.4 & $\pm \quad 1.2$ & $\begin{array}{l}+0.3 \\
-1.0 \\
\end{array}$ & $\begin{array}{l}+0.5 \\
-0.5 \\
\end{array}$ & -0.5 \\
\hline \multicolumn{6}{|c|}{$6.5<E_{T, j 1}^{*}<8.0 \mathrm{GeV}\left(<E_{T, j 1}^{*}>=7.2 \mathrm{GeV}\right)$} \\
\hline $0,0.25$ & 13.2 & $\pm \quad 0.8$ & $\begin{array}{l}+1.4 \\
+1.5 \\
\end{array}$ & $\begin{array}{l}+0.9 \\
-0.6\end{array}$ & 0.2 \\
\hline $0.25,0.375$ & 25.9 & \pm 1.5 & $\begin{array}{ll}+1.2 \\
-2.2\end{array}$ & $\begin{array}{l}+1.9 \\
{ }_{-2.0}^{+1}\end{array}$ & -0.9 \\
\hline $0.375,0.5$ & 21.9 & $\pm \quad 1.3$ & $\begin{array}{l}+1.9 \\
-1.8 \\
\end{array}$ & $\begin{array}{l}+1.6 \\
-0.9 \\
\end{array}$ & 1.9 \\
\hline $0.5,0.625$ & 18.3 & $\pm \quad 1.2$ & $\begin{array}{l}+0.8 \\
-1.0 \\
\end{array}$ & $\begin{array}{l}+1.2 \\
-1.0\end{array}$ & 0.7 \\
\hline $0.625,0.75$ & 14.8 & \pm 1.1 & $\begin{array}{ll}+0.8 \\
-0.9\end{array}$ & $\begin{array}{ll}+0.6 \\
-0.6\end{array}$ & 0.2 \\
\hline $0.75,0.875$ & 12.4 & $\pm \quad 1.0$ & $\begin{array}{l}+0.8 \\
-0.9\end{array}$ & $\begin{array}{l}+0.6 \\
-0.8\end{array}$ & 0.0 \\
\hline $0.875,1$ & 5.6 & $\pm \quad 0.7$ & $\begin{array}{l}+0.2 \\
-0.2 \\
\end{array}$ & $\begin{array}{l}+0.5 \\
-0.2 \\
\end{array}$ & -0.1 \\
\hline \multicolumn{6}{|c|}{$8.0<E_{T, j 1}^{*}<16.0 \mathrm{GeV}\left(<E_{T, j 1}^{*}>=9.7 \mathrm{GeV}\right)$} \\
\hline $0,0.25$ & 0.54 & $\pm \quad 0.05$ & $\begin{array}{l}+0.07 \\
-0.04 \\
\end{array}$ & $\begin{array}{l}+0.07 \\
-0.06\end{array}$ & 0.06 \\
\hline $0.25,0.375$ & 2.4 & $\pm \quad 0.2$ & $\begin{array}{l}+0.4 \\
-0.3\end{array}$ & $\begin{array}{l}+0.2 \\
-0.2 \\
\end{array}$ & 0.2 \\
\hline $0.375,0.5$ & 2.4 & $\pm \quad 0.2$ & $\begin{array}{l}+0.2 \\
{ }_{-0.2}^{+}\end{array}$ & $\begin{array}{l}+0.2 \\
{ }_{-0.3}^{+0.2}\end{array}$ & -0.1 \\
\hline $0.5,0.625$ & 1.9 & \pm 0.1 & $\begin{array}{l}+0.1 \\
{ }_{-0.2}\end{array}$ & $\begin{array}{l}+0.2 \\
{ }_{-0.2}\end{array}$ & 0.0 \\
\hline $0.625,0.75$ & 1.7 & $\pm \quad 0.1$ & $\begin{array}{l}+0.1 \\
-0.0 \\
\end{array}$ & $\begin{array}{l}+0.2 \\
-0.2 \\
\end{array}$ & 0.1 \\
\hline $0.75,0.875$ & 1.4 & $\pm \quad 0.1$ & $\begin{array}{l}+0.0 \\
-0.1\end{array}$ & $\begin{array}{l}+0.1 \\
-0.1 \\
\end{array}$ & 0.0 \\
\hline $0.875,1$ & 0.80 & $\pm \quad 0.09$ & $\begin{array}{l}+0.01 \\
-0.10 \\
\end{array}$ & $\begin{array}{l}+0.10 \\
-0.08 \\
\end{array}$ & -0.05 \\
\hline
\end{tabular}

Table 11: Values of the double differential cross sections with respect to $z_{\mathbb{P}}^{\mathrm{obs}}$ in bins of $E_{T, j 1}^{*}$. Other details as in the caption of Table 2. 


\begin{tabular}{|c|l|l|l|l|l|}
\hline$z_{\mathbb{P}}^{\text {obs bin }}$ & $\begin{array}{c}d \sigma / d z_{\mathbb{P}}^{\text {obs }} d Q^{2} \\
\left(\mathrm{pb} / \mathrm{GeV}^{2}\right)\end{array}$ & \multicolumn{1}{|c|}{$\begin{array}{c}\delta_{\text {stat }} \\
\left(\mathrm{pb} / \mathrm{GeV}^{2}\right)\end{array}$} & $\begin{array}{l}\delta_{\text {syst }} \\
\left(\mathrm{pb} / \mathrm{GeV}^{2}\right)\end{array}$ & $\begin{array}{l}\delta_{\mathrm{ES}} \\
\left(\mathrm{pb} / \mathrm{GeV}^{2}\right)\end{array}$ & $\begin{array}{c}\Delta_{\text {DIFFR }} \\
\left(\mathrm{pb} / \mathrm{GeV}^{2}\right)\end{array}$ \\
\hline \hline \multicolumn{6}{|c|}{$5<Q^{2}<12 \mathrm{GeV}^{2}\left(<Q^{2}>=8.1 \mathrm{GeV}^{2}\right)$} \\
\hline $0,0.25$ & 5.1 & \pm 0.3 & ${ }_{-0.5}^{+0.4}$ & ${ }_{-0.4}^{+0.4}$ & 0.0 \\
\hline $0.25,0.375$ & 8.7 & \pm 0.4 & ${ }_{-0.7}^{+0.6}$ & ${ }_{-0.7}^{+0.7}$ & -0.1 \\
\hline $0.375,0.5$ & 7.2 & \pm 0.4 & ${ }_{-0.5}^{+0.4}$ & ${ }_{-0.5}^{+0.5}$ & 0.3 \\
\hline $0.5,0.625$ & 5.2 & \pm 0.3 & ${ }_{-0.2}^{+0.1}$ & ${ }_{-0.4}^{+0.4}$ & 0.1 \\
\hline $0.625,0.75$ & 4.3 & \pm 0.3 & ${ }_{-0.2}^{+0.1}$ & ${ }_{-0.2}^{+0.3}$ & 0.1 \\
\hline $0.75,0.875$ & 2.9 & \pm 0.2 & ${ }_{-0.1}^{+0.1}$ & ${ }_{-0.2}^{+0.2}$ & -0.1 \\
\hline $0.875,1$ & 1.5 & \pm 0.2 & ${ }_{-0.2}^{+0.1}$ & ${ }_{-0.1}^{+0.1}$ & -0.1 \\
\hline \multicolumn{7}{|c|}{$12<Q^{2}<25 \mathrm{GeV}^{2}\left(<Q^{2}>=17.2 \mathrm{GeV}^{2}\right)$} \\
\hline $0,0.25$ & 1.43 & \pm 0.09 & ${ }_{-0.16}^{+0.14}$ & ${ }_{-0.07}^{+0.09}$ & 0.08 \\
\hline $0.25,0.375$ & 3.0 & \pm 0.2 & ${ }_{-0.2}^{+0.4}$ & ${ }_{-0.2}^{+0.1}$ & 0.4 \\
\hline $0.375,0.5$ & 2.3 & \pm 0.1 & ${ }_{-0.2}^{+0.1}$ & ${ }_{-0.1}^{+0.1}$ & 0.1 \\
\hline $0.5,0.625$ & 2.0 & \pm 0.1 & ${ }_{-0.2}^{+0.1}$ & ${ }_{-0.1}^{+0.1}$ & 0.0 \\
\hline $0.625,0.75$ & 1.6 & \pm 0.1 & ${ }_{-0.1}^{+0.1}$ & ${ }_{-0.1}^{+0.1}$ & 0.0 \\
\hline $0.75,0.875$ & 1.2 & \pm 0.1 & ${ }_{-0.1}^{+0.0}$ & ${ }_{-0.1}^{+0.1}$ & -0.1 \\
\hline $0.875,1$ & 0.61 & \pm 0.07 & ${ }_{-0.03}^{+0.01}$ & ${ }_{-0.03}^{+0.04}$ & -0.01 \\
\hline
\end{tabular}

Table 12: Values of the double differential cross sections with respect to $z_{\mathbb{P}}^{\mathrm{obs}}$ in bins of $E_{T, j 1}^{*}$. Other details as in the caption of Table 2. 


\begin{tabular}{|c|l|l|l|l|l|}
\hline$z_{\mathbb{P}}^{\text {obs bin }}$ & $\begin{array}{c}d \sigma / d z_{\mathbb{P}}^{\text {obs }} d Q^{2} \\
\left(\mathrm{pb} / \mathrm{GeV}^{2}\right)\end{array}$ & $\begin{array}{c}\delta_{\text {stat }} \\
\left(\mathrm{pb} / \mathrm{GeV}^{2}\right)\end{array}$ & $\begin{array}{l}\delta_{\text {syst }} \\
\left(\mathrm{pb} / \mathrm{GeV}^{2}\right)\end{array}$ & $\begin{array}{l}\delta_{\mathrm{ES}} \\
\left(\mathrm{pb} / \mathrm{GeV}^{2}\right)\end{array}$ & $\begin{array}{c}\Delta_{\text {DIFFR }} \\
\left(\mathrm{pb} / \mathrm{GeV}^{2}\right)\end{array}$ \\
\hline \hline \multicolumn{6}{|c|}{$25<Q^{2}<50 \mathrm{GeV}^{2}\left(<Q^{2}>=35.2 \mathrm{GeV}^{2}\right)$} \\
\hline $0,0.25$ & 0.51 & \pm 0.04 & ${ }_{-0.08}^{+0.03}$ & ${ }_{-0.02}^{+0.03}$ & -0.06 \\
\hline $0.25,0.375$ & 1.03 & \pm 0.07 & ${ }_{-0.13}^{+0.07}$ & ${ }_{-0.07}^{+0.06}$ & -0.03 \\
\hline $0.375,0.5$ & 1.00 & \pm 0.07 & ${ }_{-0.06}^{+0.06}$ & ${ }_{-0.06}^{+0.04}$ & 0.05 \\
\hline $0.5,0.625$ & 0.77 & \pm 0.06 & ${ }_{-0.02}^{+0.06}$ & ${ }_{-0.04}^{+0.04}$ & 0.06 \\
\hline $0.625,0.75$ & 0.60 & \pm 0.05 & ${ }_{-0.04}^{+0.03}$ & ${ }_{-0.03}^{+0.04}$ & -0.01 \\
\hline $0.75,0.875$ & 0.44 & \pm 0.04 & ${ }_{-0.02}^{+0.01}$ & ${ }_{-0.04}^{+0.03}$ & 0.00 \\
\hline $0.875,1$ & 0.24 & \pm 0.03 & ${ }_{-0.01}^{+0.01}$ & ${ }_{-0.01}^{+0.02}$ & 0.00 \\
\hline \multicolumn{7}{|c|}{$50<Q^{2}<100 \mathrm{GeV}^{2}\left(<Q^{2}>=69.5 \mathrm{GeV}^{2}\right)$} \\
\hline 0.0 .01 \\
\hline $0.25,0.375$ & 0.25 & \pm 0.01 & ${ }_{-0.01}^{+0.00}$ & ${ }_{-0.01}^{+0.01}$ & 0.00 \\
\hline $0.375,0.5$ & 0.28 & \pm 0.02 & ${ }_{-0.01}^{+0.05}$ & ${ }_{-0.01}^{+0.02}$ & 0.05 \\
\hline $0.5,0.625$ & 0.20 & \pm 0.02 & ${ }_{-0.01}^{+0.02}$ & ${ }_{-0.01}^{+0.01}$ & 0.02 \\
\hline $0.625,0.75$ & 0.16 & \pm 0.02 & ${ }_{-0.00}^{+0.01}$ & ${ }_{-0.01}^{+0.01}$ & 0.01 \\
\hline $0.75,0.875$ & 0.13 & \pm 0.02 & ${ }_{-0.00}^{+0.00}$ & ${ }_{-0.01}^{+0.01}$ & 0.00 \\
\hline $0.875,1$ & 0.11 & \pm 0.02 & ${ }_{-0.02}^{+0.01}$ & ${ }_{-0.01}^{+0.01}$ & -0.01 \\
\hline
\end{tabular}

Table 13: Values of the double differential cross sections with respect to $z_{\mathbb{P}}^{\text {obs }}$ in bins of $E_{T, \mathrm{j} 1}^{*}$. Other details as in the caption of Table 2. 


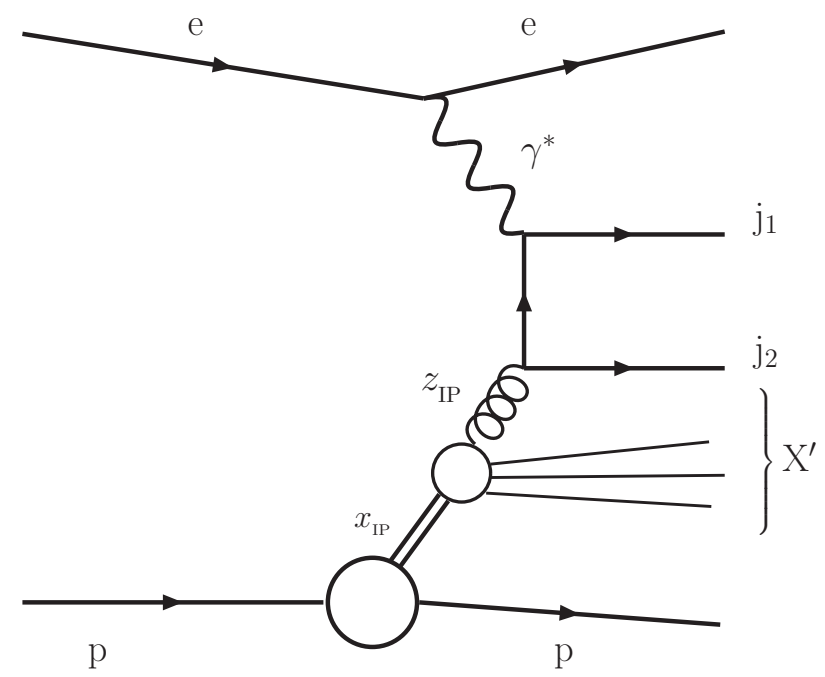

Figure 1: Schematic representation of the boson-gluon fusion diagram for LO dijet production in diffractive DIS. 


\section{ZEUS}

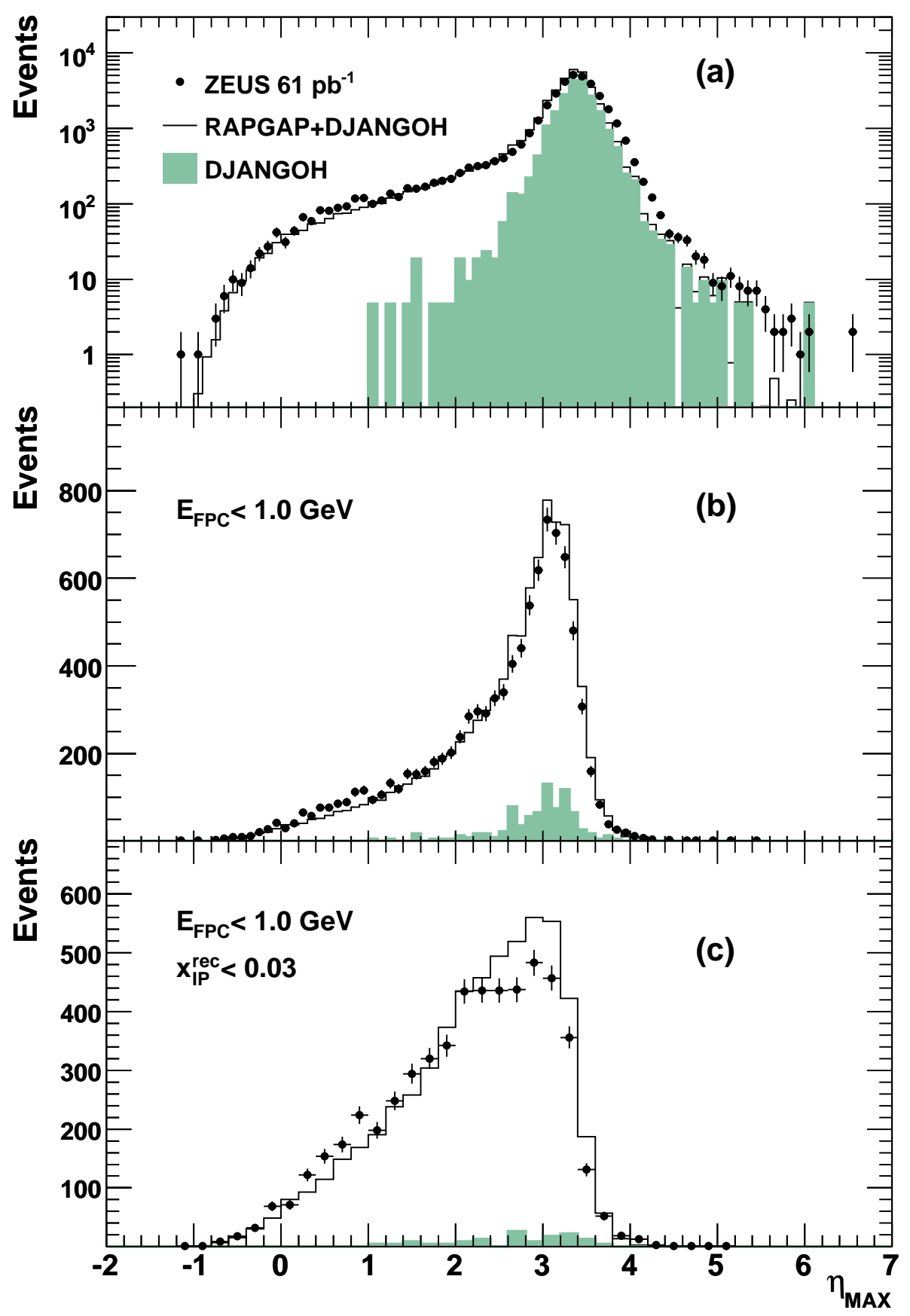

Figure 2: The measured $\eta_{\text {MAX }}$ distribution (dots) (a) before diffractive selection, (b) after the $E_{F P C}$ cut and (c) after adding the $x_{\mathbb{P}}^{\mathrm{obs}}$ cut. Also shown are areanormalised $M C$ expectations obtained by fitting the relative amount of RAPGAP and DJANGOH to give the best description of the data before any diffractive selection. 


\section{ZEUS}
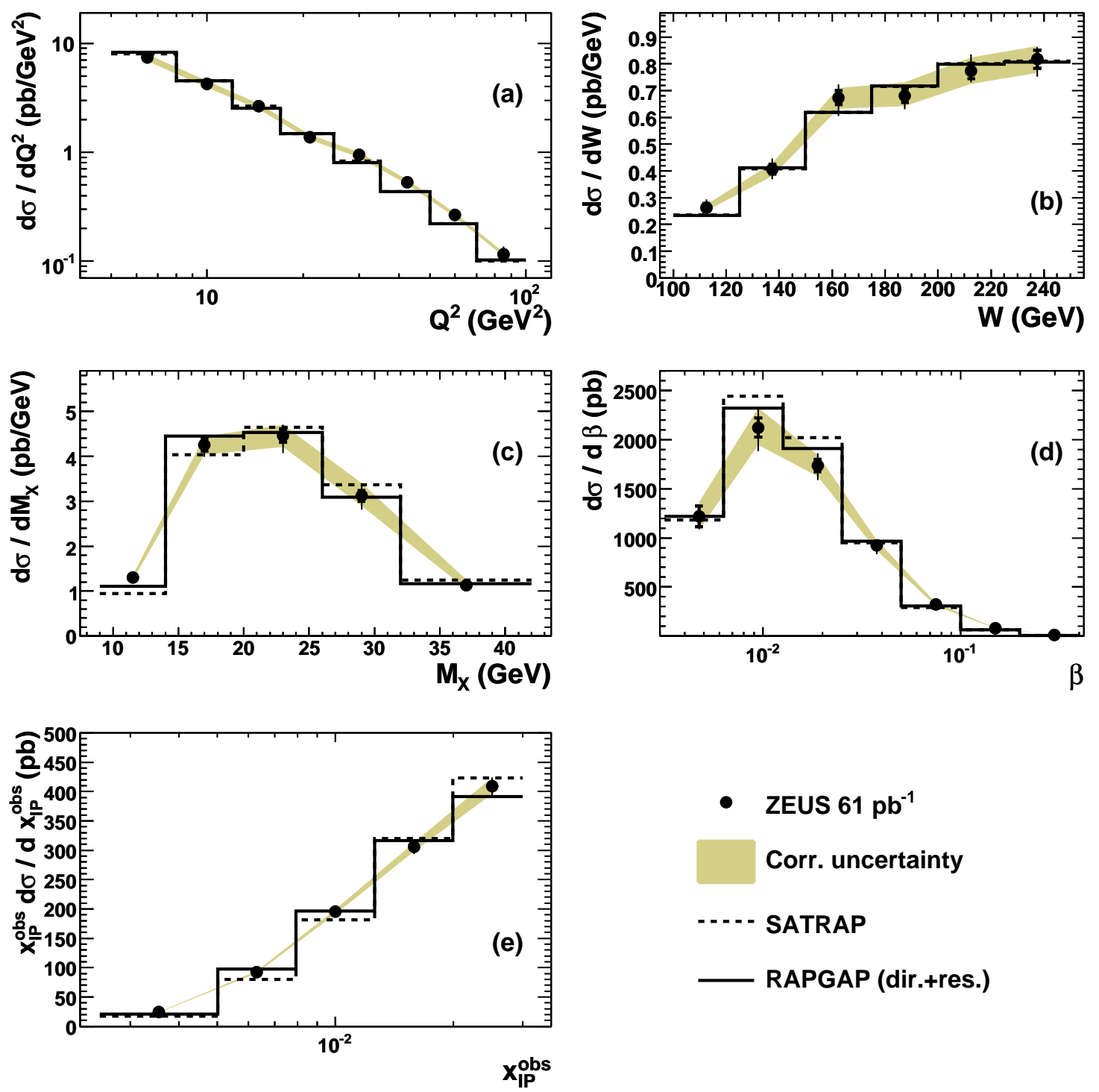

- ZEUS $61 \mathrm{pb}^{-1}$

Corr. uncertainty

SATRAP

RAPGAP (dir.+res.)

Figure 3: Measured differential cross section (dots) as a function of (a) $Q^{2}$, (b) $W,(c) M_{X},(d) \beta$ and (e) $x_{\mathbb{P}}^{\mathrm{obs}}$. The inner error bars represent the statistical uncertainty and the outer error bars represent the statistical and uncorrelated systematic uncertainties added in quadrature. The shaded band represents the correlated uncertainty. For comparison the area-normalised predictions of the RAPGAP (solid lines) and the SATRAP (dashed lines) MC models are also shown. 


\section{ZEUS}
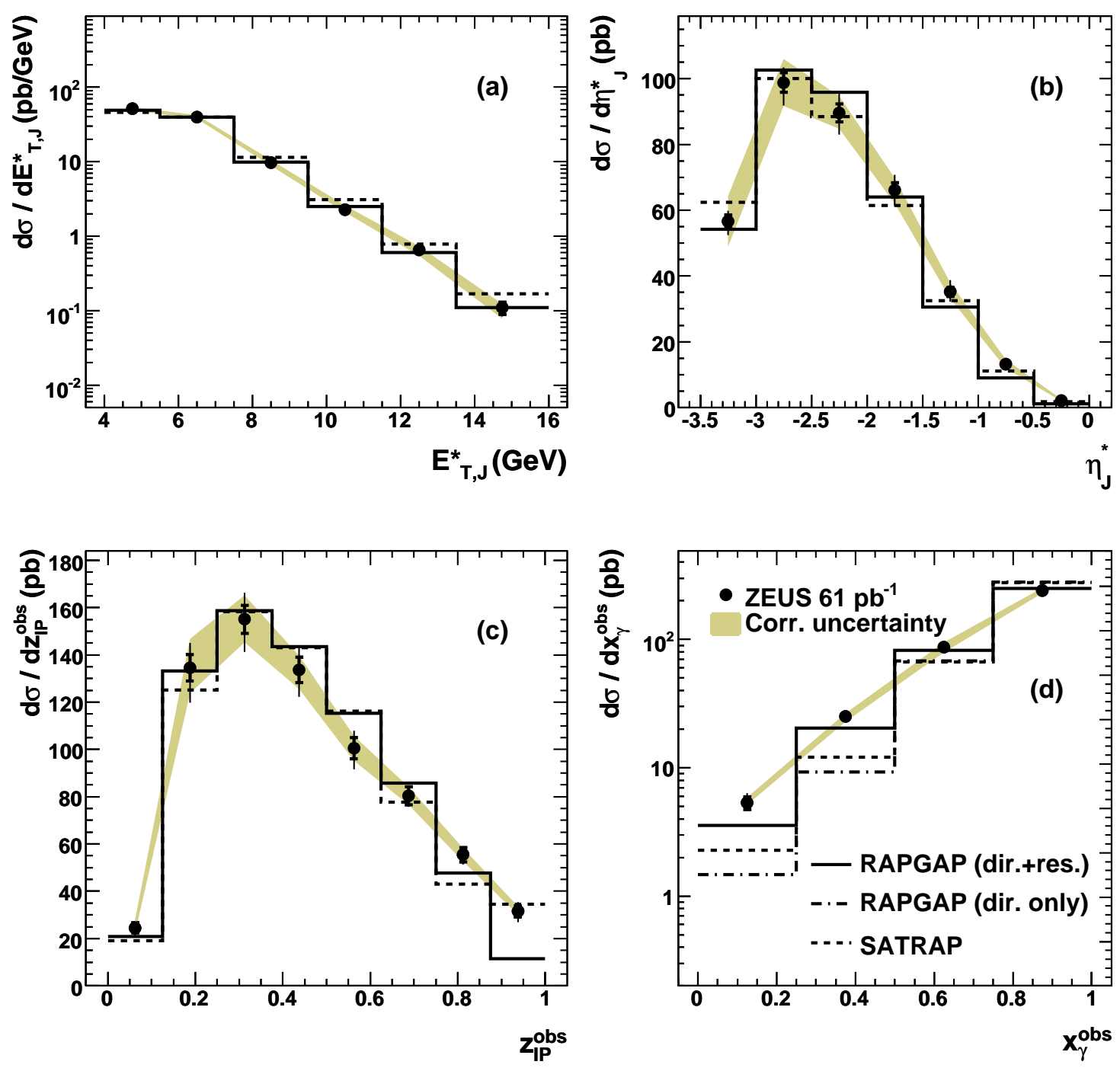

Figure 4: Measured differential cross section as a function of (a) $E_{\mathrm{T}, \mathrm{J}}^{*}$, (b) $\eta_{\mathrm{J}}^{*},(c)$ $z_{\mathbb{P}}^{\mathrm{obs}}$ and $(d) x_{\gamma}^{\mathrm{obs}}$. The dashed-dotted line represents the area-normalised RAPGAP with only the direct photon contribution. Other details as in the caption of Fig. 3. 


\section{ZEUS}
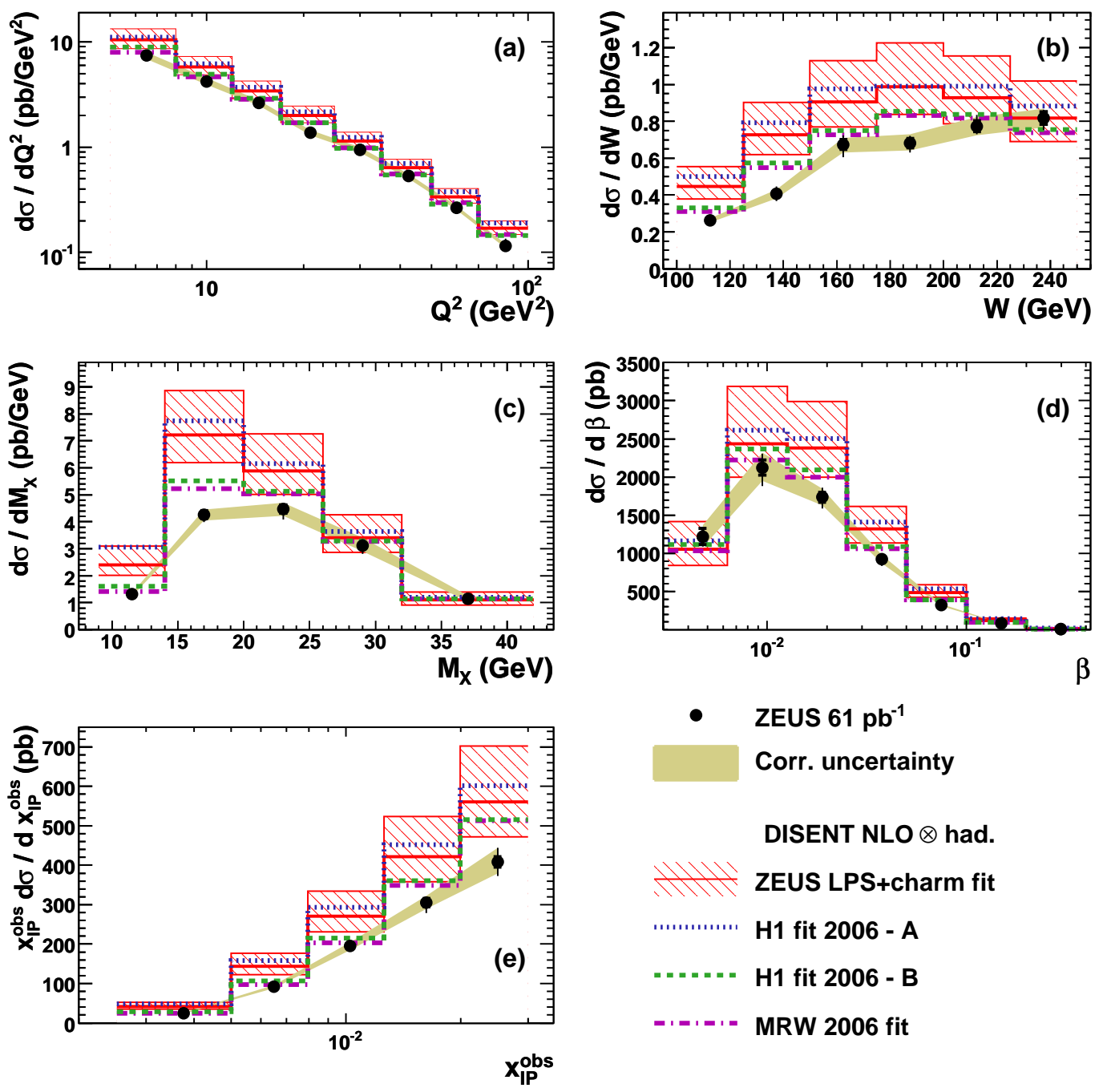

- ZEUS $61 \mathrm{pb}^{-1}$

Corr. uncertainty

DISENT NLO $\otimes$ had.

ZEUS LPS+charm fit

H1 fit 2006 - A

H1 fit 2006 - B

- - = - - MRW $2006 \mathrm{fit}$

Figure 5: Measured differential cross section as a function of (a) $Q^{2}$, (b) $W$, (c) $M_{X}$, (d) $\beta$ and (e) $x_{\mathbb{P}}^{\text {obs }}$ compared to the NLO predictions obtained using the available dPDFs, as indicated in the figure. The hatched area indicates the theoretical uncertainty of the predictions estimated using the ZEUS LPS+charm dPDFs. Other details as in the caption of Fig. 3. 


\section{ZEUS}
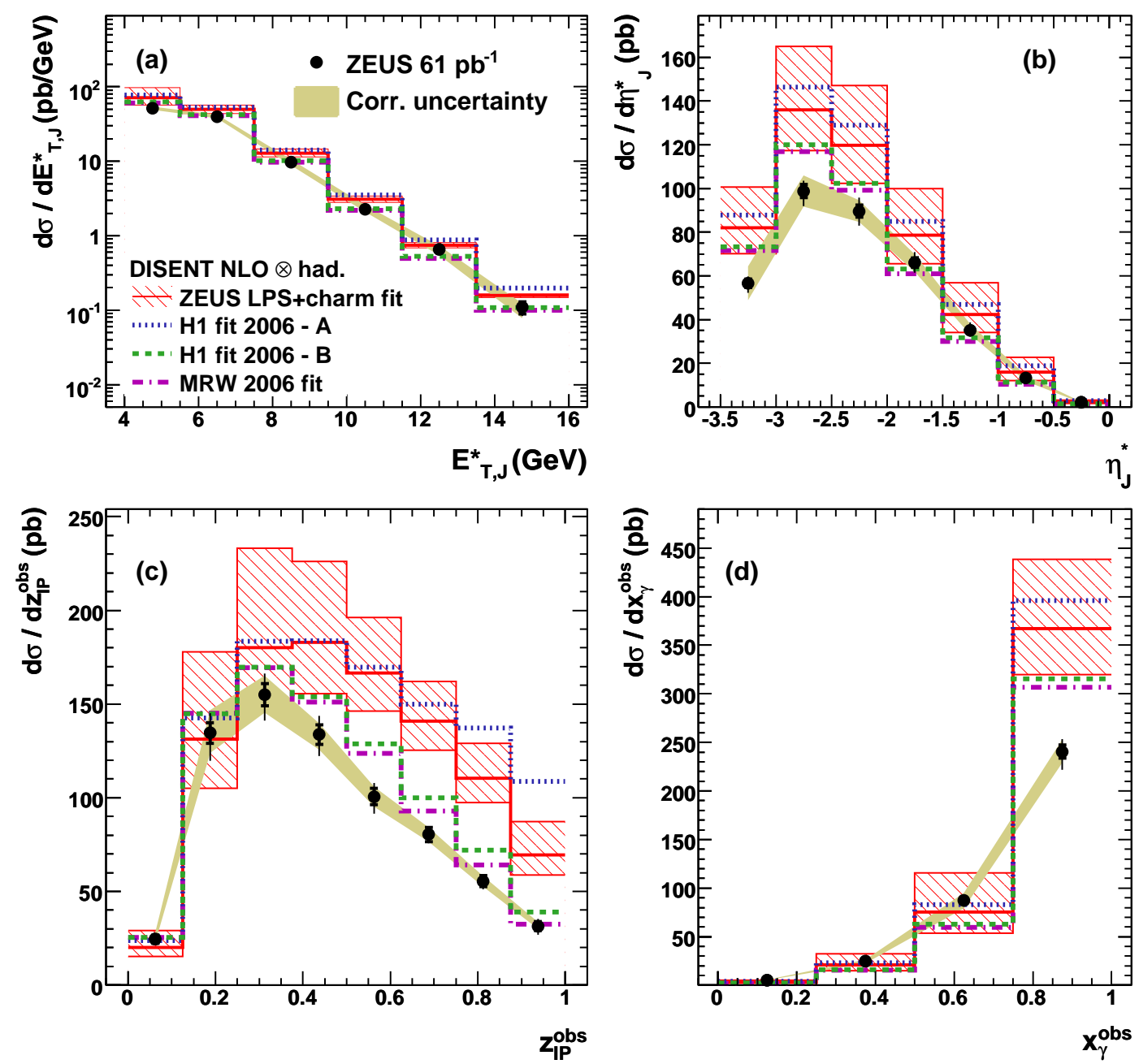

Figure 6: Measured differential cross section as a function of (a) $E_{\mathrm{T}, \mathrm{J}}^{*}$, (b) $\eta_{\mathrm{J}}^{*}$, (c) $z_{\mathbb{P}}^{\mathrm{obs}}$ and (d) $x_{\gamma}^{\mathrm{obs}}$ compared to the NLO prediction obtained using the available dPDFs. Other details as in the caption of Fig. 5. 


\section{ZEUS}
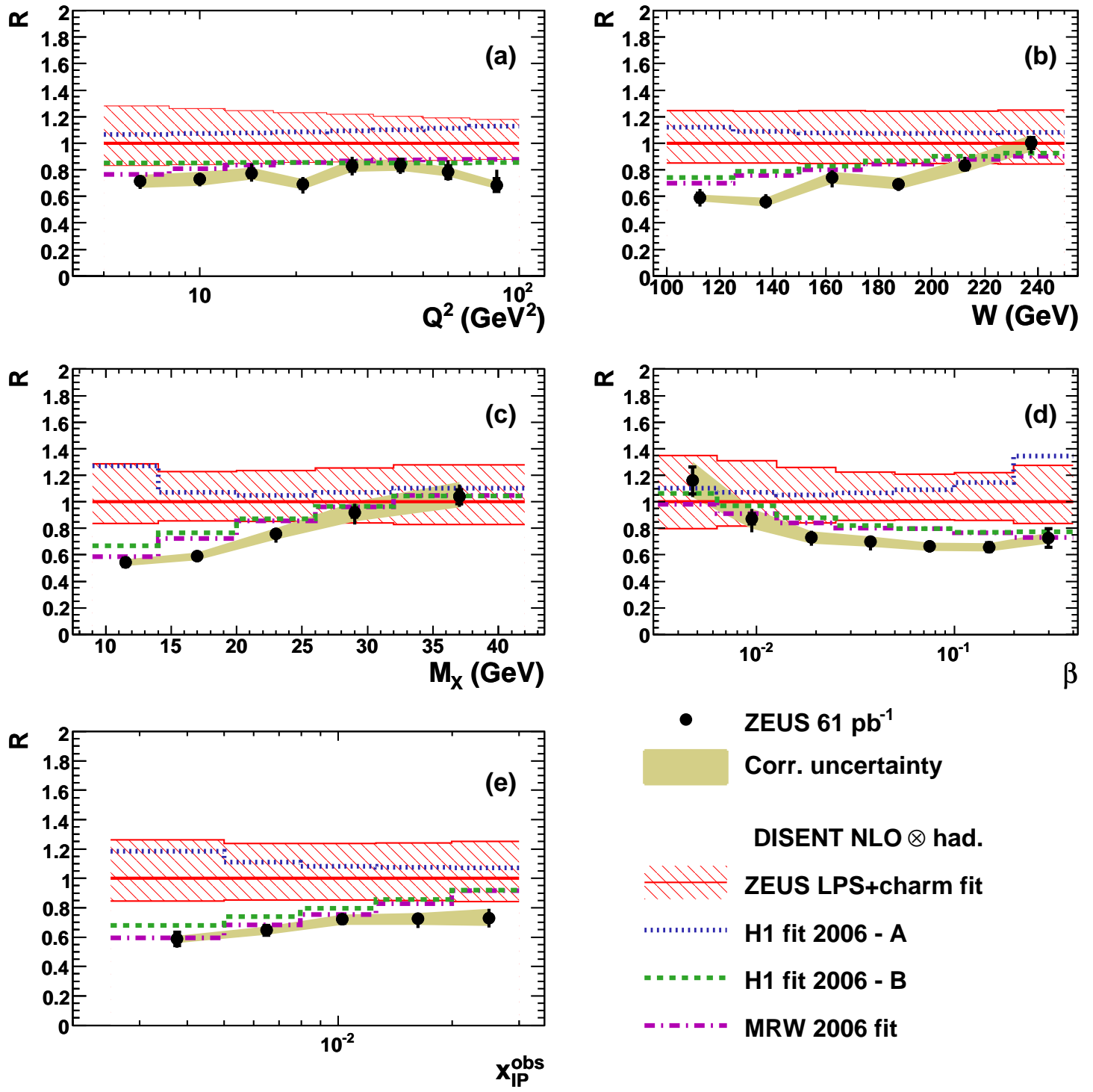

- ZEUS $61 \mathrm{pb}^{-1}$

Corr. uncertainty

DISENT NLO $\otimes$ had.

\section{ZEUS LPS+charm fit}

H1 fit 2006 - A

H1 fit 2006 - B

MRW 2006 fit

Figure 7: Ratio, R, of the data to the NLO prediction using the ZEUS LPS+charm dPDFs (dots) as function of (a) $Q^{2}$, (b) $W$, (c) $M_{X}$, (d) $\beta$ and (e) $x_{\mathbb{P}}^{\text {obs }}$. Also shown is the ratio of NLO calculations with other dPDFs to ZEUS LPS+charm. Other details as in the caption of Fig. 5. 


\section{ZEUS}
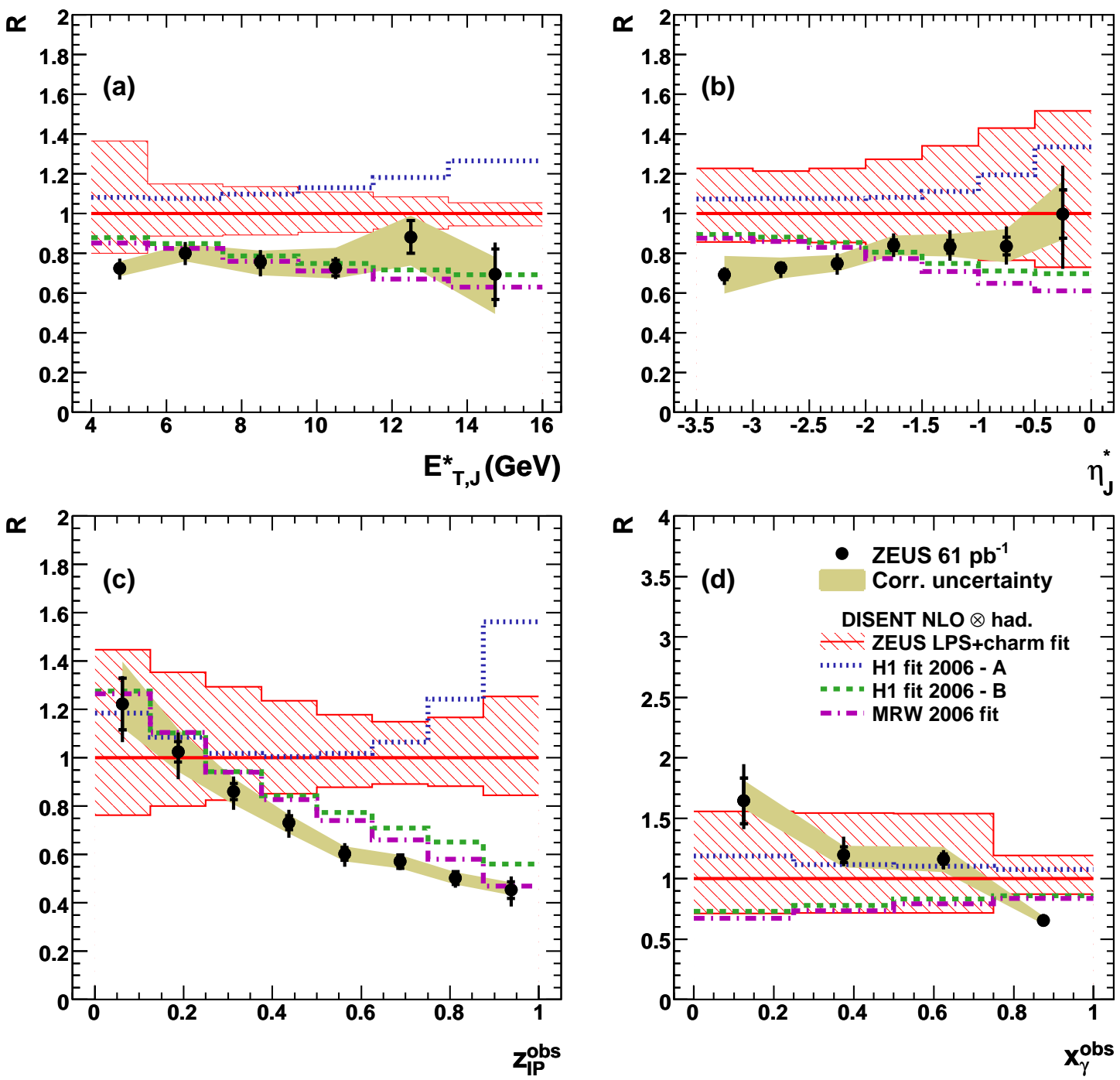

Figure 8: Ratio, R, of the data to the NLO prediction using the ZEUS LPS+charm dPDFs (dots) as function of (a) $E_{\mathrm{T}, \mathrm{J}}^{*},(b) \eta_{\mathrm{J}}^{*}$, (c) $z_{\mathbb{P}}^{\mathrm{obs}}$ and (d) $x_{\gamma}^{\mathrm{obs}}$. Other details as in the caption of Fig. $\%$. 


\section{ZEUS}
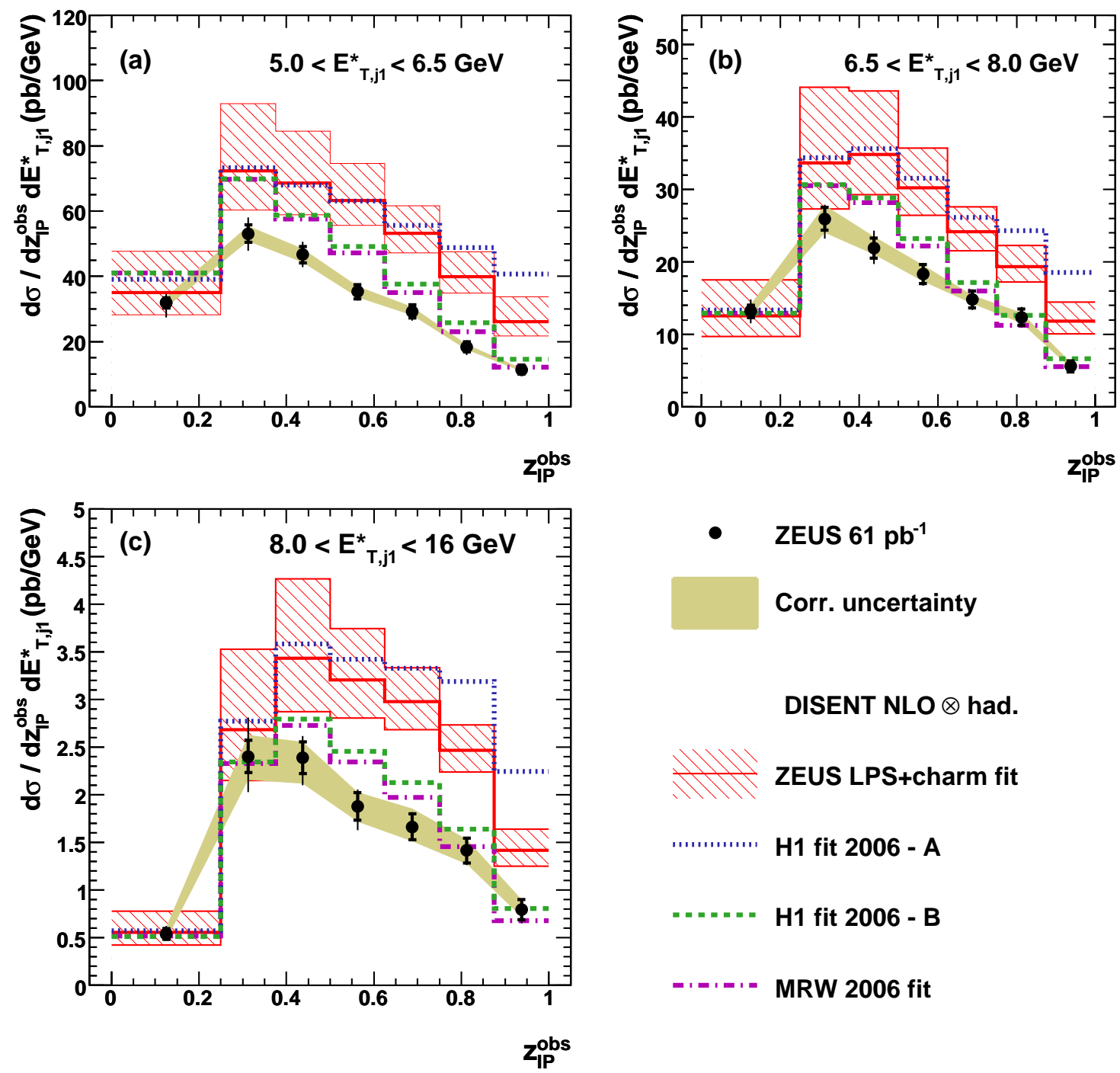

- ZEUS $61 \mathrm{pb}^{-1}$

Corr. uncertainty

DISENT NLO $\otimes$ had.

ZEUS LPS+charm fit

H1 fit 2006 - A

H1 fit 2006 - B

MRW 2006 fit

Figure 9: $\quad$ Measured differential cross section as a function of $z_{\mathbb{P}}^{\text {obs }}$ in different regions of $E_{T, j 1}^{*}$ (dots). Other details as in the caption of Fig. 5. 


\section{ZEUS}
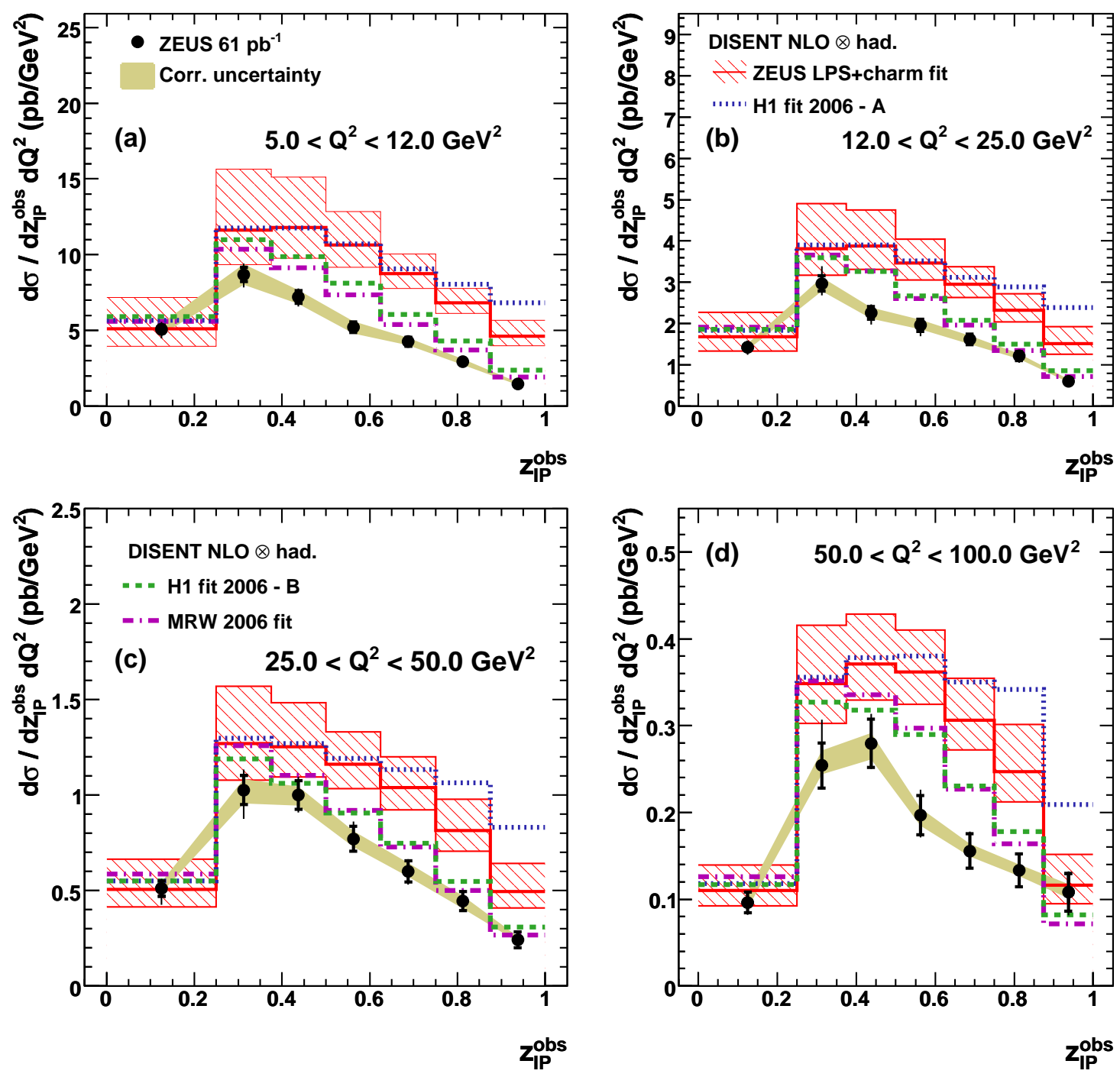

Figure 10: Measured differential cross section as a function of $z_{\mathbb{P}}^{\text {obs }}$ in different regions of $Q^{2}$ (dots). Other details as in the caption of Fig. 5. 